\title{
Dosimetry tools and techniques for IMRT
}

\author{
Daniel A. Low ${ }^{\text {a) }}$ \\ Washington University, St. Louis, Missouri 63110 \\ Jean M. Moran \\ University of Michigan, Ann Arbor, Michigan 48109 \\ James F. Dempsey \\ Viewray Incorporated, Cleveland, Ohio 44106 \\ Lei Dong \\ M. D. Anderson Cancer Center, Houston, Texas 77013 \\ Mark Oldham \\ Duke University Medical Center, Durham, North Carolina 27710
}

(Received 25 January 2010; revised 4 October 2010; accepted for publication 4 October 2010; published 16 February 2011)

\begin{abstract}
Intensity modulated radiation therapy (IMRT) poses a number of challenges for properly measuring commissioning data and quality assurance (QA) radiation dose distributions. This report provides a comprehensive overview of how dosimeters, phantoms, and dose distribution analysis techniques should be used to support the commissioning and quality assurance requirements of an IMRT program. The proper applications of each dosimeter are described along with the limitations of each system. Point detectors, arrays, film, and electronic portal imagers are discussed with respect to their proper use, along with potential applications of 3D dosimetry. Regardless of the IMRT technique utilized, some situations require the use of multiple detectors for the acquisition of accurate commissioning data. The overall goal of this task group report is to provide a document that aids the physicist in the proper selection and use of the dosimetry tools available for IMRT QA and to provide a resource for physicists that describes dosimetry measurement techniques for purposes of IMRT commissioning and measurement-based characterization or verification of IMRT treatment plans. This report is not intended to provide a comprehensive review of commissioning and QA procedures for IMRT. Instead, this report focuses on the aspects of metrology, particularly the practical aspects of measurements that are unique to IMRT. The metrology of IMRT concerns the application of measurement instruments and their suitability, calibration, and quality control of measurements. Each of the dosimetry measurement tools has limitations that need to be considered when incorporating them into a commissioning process or a comprehensive QA program. For example, routine quality assurance procedures require the use of robust field dosimetry systems. These often exhibit limitations with respect to spatial resolution or energy response and need to themselves be commissioned against more established dosimeters. A chain of dosimeters, from secondary standards to field instruments, is established to assure the quantitative nature of the tests. This report is intended to describe the characteristics of the components of these systems; dosimeters, phantoms, and dose evaluation algorithms. This work is the report of AAPM Task Group 120. (C) 2011 American Association of Physicists in Medicine. [DOI: 10.1118/1.3514120]
\end{abstract}

Key words: radiation therapy, dosimetry, intensity modulated radiation therapy, quality assurance, metrology, radiotherapy

\section{TABLE OF CONTENTS}

\section{DIFFERENCES IN DOSIMETRY REQUIREMENTS BETWEEN} CONVENTIONAL RADIATION THERAPY AND

IMRT. ........................ 1314

I.A. Dose distribution complexity.......... 1314

I.B. Temporal nature of IMRT dose delivery and its impact on absolute dosimetry......... 1314

I.C. Monitor units. . . . . . . . . . . . . 1315

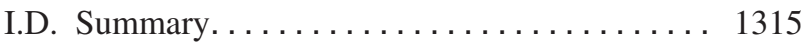

II. DOSIMETERS. . . . . . . . . . . . . . . . 1315
II.A. Point dosimeters. . . . . . . . . . . . 1315

II.A.1. Ionization chambers. . . . . . . . . . . . 1315

II.A.2. Solid state dosimeters. . . . . . . . . . . 1317

II.A.3. Electrometer and cable performance...... 1318

II.A.4. Applications to IMRT. . . . . . . . . . 1319

II.A.5. Summary of recommendations. . . . . . . 1320

II.B. Two-dimensional dosimetry. . . . . . . . . . . 1321

II.B.1. Film. . . . . . . . . . . . . . . . 1321

II.B.2. Array detectors. . . . . . . . . . . . . . . 1324

II.B.3. Computed radiography. . . . . . . . . . . 1325

III. PHANTOMS. . . . . . . . . . . . . . . . . . . . . 1325

III.A. Phantom types................... 1325 
III.A.1. Phantom selection for IMRT. .......... 1325

III.A.2. Geometric phantoms............... 1325

III.A.3. Anthropomorphic phantoms. . . . . . . . . 1326

III.B. Phantom materials. . . . . . . . . . . . . . . 1326

III.C. Phantom characterization. . . . . . . . . . . . . 1326

IV. DOSE DISTRIBUTION COMPARISONS AND

EVALUATIONS. . . . . . . . . . . . . . . . . . 1326

IV.A. Dose distribution registration. . . . . . . . 1326

IV.B. Dose comparison tools............... 1327

IV.B.1. Dose distribution overlays........... 1327

IV.B.2. Dose-difference distributions. . . . . . . . . . 1327

IV.B.3. Distance-to-agreement. . .............. 1328

IV.B.4. Quantitative comparison tools.......... 1329

IV.C. Summary..................... 1330

V. ADDITIONAL SYSTEMS FOR QUALITY

ASSURANCE....................... 1330

V.A. Electronic portal imaging devices....... 1330

V.A.1. Application to IMRT.............. 1331

V.A.2. Recommendations for use........... 1331

V.B. Three-dimensional detectors. ........... 1331

V.B.1. Polymerizing gels.............. 1331

V.B.2. Fricke and radiochromic gels and plastics. 1332

V.B.3. Readout techniques.............. 1332

V.B.4. Recommendations for use........... 1332

VI. SUMMARY........................ 1332

\section{DIFFERENCES IN DOSIMETRY REQUIREMENTS BETWEEN CONVENTIONAL RADIATION THERAPY AND IMRT}

\section{I.A. Dose distribution complexity}

The complex three-dimensional (3D) shapes of intensity modulated radiation therapy (IMRT) dose distributions and the methods for IMRT dose delivery demand that the dosimetry measurement techniques typically employed for conventional three-dimensional conformal radiation therapy (3DCRT), including straightforward dynamic techniques such as conformal arc and dynamic wedge, be reviewed and adapted for the unique challenges posed by IMRT. The complex shape of the dose distribution leads to numerous regions containing steep dose gradients, even within target volumes. Understanding the limitations and use of dosimeters to measure these dose distributions is critical to safe IMRT implementation.

Conventional 3DCRT treatment planning typically utilizes a beam's eye view approach. Validation of 3DCRT treatment plans can be accomplished utilizing traditional dosimetry techniques, such as scanning ionization chambers and monitor unit (MU) verification calculations, because these plans are composed of superpositions of relatively large, low fluence gradient, radiation segments. However, IMRT treatments involve the delivery of complex dose distribution shapes that place steep dose gradients near critical structures in an optimized 3D configuration. The use of fluence modulation allows the radiation beam orientations to be decoupled from the tumor and critical structure geometries so that radiation beams can be aimed directly through critical struc- tures and the fluence modulation optimization process will limit the critical structure doses. Unlike 3DCRT, in IMRT portal images cannot be used to validate critical structure avoidance. The 3D complexity of the dose, along with the decoupling of the beam geometry and the resulting dose, means that the quality assurance (QA) of IMRT dose distributions needs to concentrate more on the cumulative delivered dose rather than on the QA of individual segments, as well as checking the dose at multiple locations.

The dose gradients in the IMRT dose distributions are intended to match the patient's target and normal organ geometry. Not only are the relative gradient positions important to validate, but the absolute positions also need to be verified. The IMRT dose QA measurements need to explicitly include a quantitative registration process for independently validating the spatial location of the dose gradients. Such a registration process could involve the use of fixed landmarks on the phantom that are colocated with radiopaque markers such that alignment of the phantom to the linear accelerator coordinate system using the positioning lasers can be registered with the alignment of the phantom in the treatment planning system.

\section{I.B. Temporal nature of IMRT dose delivery and its impact on absolute dosimetry}

In most 3DCRT procedures, the radiation beam is static, meaning that beam modifiers and the gantry, collimator, and couch remain stationary during irradiation. Because the radiation beam is static, characterization of the radiation beam can be conducted by scanning ionization chambers in water phantoms, using interpolation when necessary to fill in the spaces between the measured points. The dosimetric accuracy of ionization chambers allows for a quantitative measurement of the radiation beam. While it is impractical during 3DCRT planning system commissioning to measure every clinically used beam configuration, the measurement of a subset of beams is sufficient. ${ }^{1}$ IMRT dose delivery is often a dynamic process where the incident fluence shape and intensity are varying during the treatment, so scan-based dose measurements become impractical. Therefore, dose measurements of IMRT treatment plans are limited to integrating dosimetric techniques.

Absolute dosimeters are defined here as those whose results, for absorbed dose to water, require no adjustment or renormalization other than those done in accordance with the established dosimetry protocols (e.g., TG 51). As an example, a small-volume ionization chamber is typically crosscalibrated, in the beam quality in question, against an ionization chamber with a NIST-traceable calibration. The crosscalibrated ionization chamber is then used to measure absorbed dose (e.g., in Gy) without the need for any renormalization (other than for temperature and pressure corrections). Factors such as nonuniformity, energy dependence, and response instability exclude some dosimetry systems from being used for absolute dosimetry. Recommendations for the specific use of dosimetry systems are provided in this report. 


\section{I.C. Monitor units}

Computational MU checks of 3DCRT treatment plans are the standard of practice for MU validation. These MU checks often use portal shape approximations to determine the required photon radiation scatter parameters. In conventional 3DCRT, these parameters change slowly with respect to the field size and depth, and field-dependent approximations yield MU calculations that are accurate to within a few percent. ${ }^{2,3}$ This accuracy is considered adequate by the radiation therapy community and is one method used to avoid catastrophic clinical errors.

For IMRT dose delivery sequences, either dynamic or static, the dose at any given location within the target volume is delivered by a fraction of the total segments in a field. The number of MUs required to deliver the dose distribution will be a strong function of a complex leaf delivery sequence and is therefore unpredictable based on only the target dose and patient geometry. A qualified medical physicist can no longer judge whether the number of MUs is correct based on professional knowledge and experience, so catastrophic errors may not be caught without an independent, quantitative validation mechanism. Either direct dose measurements, conducted using phantoms, or independent dose calculation software, is required to validate the treatment planning output on a patient-specific basis. Independent dose calculation methods (sometimes called monitor unit calculation methods) have been developed and are being used in many centers as a component of the patient-specific QA program. ${ }^{4-15}$ Dose calculation methods will continue to evolve and their role in patient-specific QA is likely to expand. One limitation of such independent dose calculation methods is that the deliverability of the IMRT plan is not validated on the actual treatment device. For this reason, patient-specific validation is often conducted using direct dose distribution measurements in homogeneous solid media.

\section{I.D. Summary}

The greater need for dose-measurement-based validation, coupled with their greater dose distribution complexity and the temporal nature of dose delivery, led to this review of dose measurement systems for IMRT. In this report, we will describe the uses and pitfalls of the most relevant dosimetry systems and highlight the unique influence of IMRT on their use. We will also provide recommendations for proper operations of specific dosimeters.

There are also other task group reports that are relevant to the subject of IMRT dosimetry. The Task Group 106 of the AAPM (Ref. 16) recently published a report entitled "Accelerator beam data commissioning equipment and procedures: Report of the TG-106 of the Therapy Physics Committee of the AAPM" that provided recommendations for detector selection. The Task Group 119 (Ref. 17) of the AAPM published a report comparing different IMRT commissioning results using a standard treatment plan dataset and Task Group $142,{ }^{18}$ the QA of medical linear accelerators, provides IMRT $\mathrm{QA}$ recommendations.
This report includes examples of commercial products. This should not be considered an endorsement by this task group or the AAPM.

\section{DOSIMETERS}

\section{II.A. Point dosimeters}

\section{II.A.1. Ionization chambers}

Cylindrical ionization chambers are used for point-dose measurements in megavoltage photon radiation therapy because of their excellent stability, linear response to absorbed dose, small directional dependence, beam-quality response independence, and traceability to a primary calibration standard. High-spatial resolution is important for IMRT measurements. ${ }^{14,19-30}$ Table I lists some of the commercially available "small" volume ionization chambers and their design characteristics according to the manufacturer's literature. All listed chambers are waterproof and designed with air-ventilation. All of these ionization chambers have cylindrical symmetry, minimizing the sensitivity response variation as a function of beam entry angle when the beam central axis is perpendicular to the chamber axis of symmetry. In Table I, the effective length is the length of the active volume along the axis and the effective diameter is in the radial direction perpendicular to the axis. Unfortunately, there have been a few published studies on the oblique-incidence response of cylindrical ionization chambers to megavoltage beams. If the dose distribution verification measurements include noncoplanar beams, the physicist should determine the sensitivity of the ionization chamber in such geometries.

II.A.1.a. Volume averaging. All ionization chambers exhibit some amount of volume averaging. This is due to the fact that the collected ions are created throughout the active chamber volume. Volume averaging will cause perturbations in dose distribution measurements in regions with high curvature (large second-order spatial derivatives in the dose distributions). These typically occur in conjunction with regions of steep dose gradients, so the effect of volume averaging is typically associated with beam penumbra regions. Bouchard and Seuntjens ${ }^{25}$ recently examined the perturbations in ionization chamber reference dosimetry for IMRT beams and provided a methodology for correcting the chamber response to account for nonuniform dose distributions. Monte Carlo dose calculations were used to determine perturbation factors for cylindrical ionization chambers. Perturbation factors for relatively large ionization chambers (Farmer, $6 \mathrm{~mm}$ in diameter, $23 \mathrm{~mm}$ length active volume) in individual static and dynamic IMRT fields were determined and found to exceed $10 \%$ in some cases. ${ }^{25}$ The sensitivity (dose to the collection air cavity per incident photon) was sharply peaked at the projected ionization chamber cavity volume boundaries. The nonuniform dose response, coupled with the heterogeneous photon beam fluence, was responsible for the nonunity IMRT correction factor. They concluded that fluence-perturbation correction factors should be employed for IMRT dose measurements. A second conclusion was that one should assure that the ionization chamber be placed in regions of relatively 


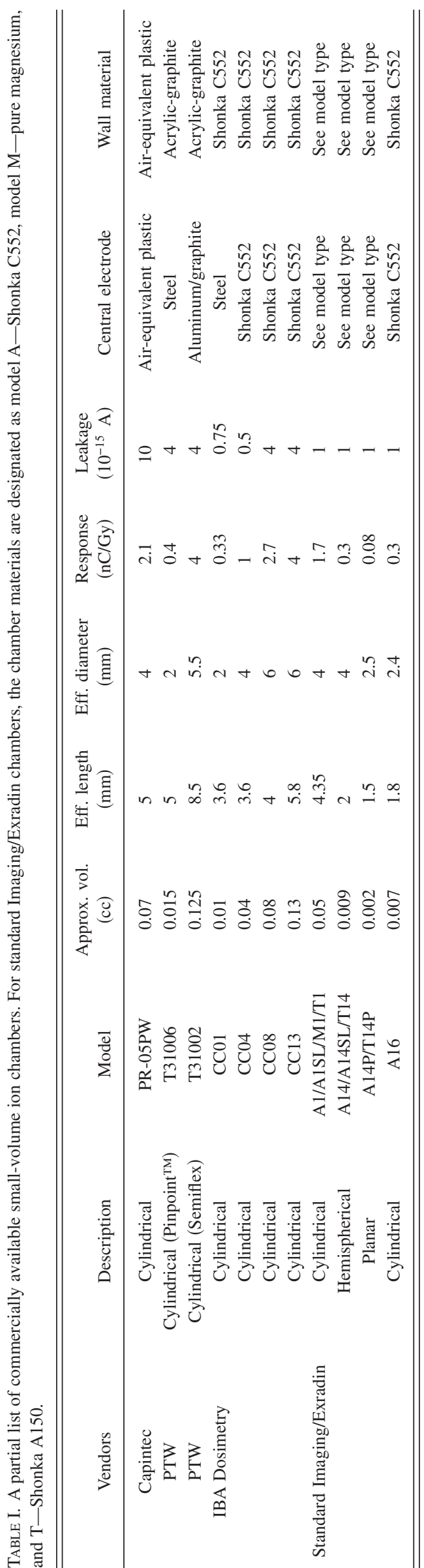

homogeneous fluence and that the relative size of the ionization chamber and homogeneous fluence region be determined prior to the measurement. For measurements that check the total delivered dose, the ionization chamber should be placed in regions of homogeneous total dose.

While Bouchard and Seuntjens ${ }^{25}$ showed that ionization chamber measurements can yield substantial dose measurement errors for IMRT beams, Low et al. ${ }^{24}$ showed that even large ionization chambers will accurately integrate dose across homogenous IMRT dose regions. Based on these results, we conclude that there are two main clinical scenarios where volume averaging becomes a concern. First, when measuring the output at the center of a small static field, volume averaging will result in an underestimate of the actual dose. In this case, care needs to be taken to assure that the ionization chamber is sufficiently small to accurately measure the dose, or another, high-spatial resolution dosimeter be used to conduct the measurement. Second, when measuring the profile of dose distributions with rapidly spatially varying doses, the measured shape of the dose distribution will be blurred by volume averaging. If an accurate measurement of the profile shape is important, a higher spatial resolution detector will be required.

Regarding the size of the ionization chamber, if the treatment planning system has the capability of computing the average dose throughout the active ionization chamber volume, the use of a larger ionization chamber will reduce the sensitivity of the measurement to positioning inaccuracies. A small ionization chamber dose measurement will be more sensitive to positioning errors. However, this should not be taken as a recommendation to place a large ionization chamber in a high-dose homogenous region that is smaller than the active chamber volume. If the treatment planning system cannot provide the volume-averaged dose, a small ionization chamber should be used for the measurements, and the chamber should be placed in a region of shallow dose gradient.

II.A.1.b. Energy response. The energy response of modern ionization chambers is flat for megavoltage photon beam dosimetry. However, for some very small chambers, in order to increase the ionization signal, the central electrode is made of high- $Z$ materials, causing significant sensitivity variations with field size and depth. For example, the central collecting electrode is made of steel in both the PTW PinPoint ${ }^{\mathrm{TM}}$ and the Wellhofer/Scanditronix CC01 chambers. This causes over-response to low-energy scattered photons, ${ }^{20}$ which are abundant in large treatment fields or under heavily blocked areas. Consequently, care should be taken when using steelelectrode ionization chambers for IMRT dose distribution measurements. While this problem is not specific to IMRT dose distribution measurements, characterizing and accounting for the variation in sensitivity to low-energy scattered photons is more critical for IMRT than for static open fields.

II.A.1.c. Ionization chamber stability. A properly functioning ionization chamber should demonstrate stable response over time. A quality assurance program that crosschecks the ionization chamber sensitivity against a local secondary standard ionization chamber should be employed. 
TABLE II. Summary of a few commercially available solid state detectors.

\begin{tabular}{|c|c|c|c|c|c|c|c|}
\hline Vendors & Description & Model & $\begin{array}{l}\text { Approx. vol. } \\
\text { (cc) }\end{array}$ & $\begin{array}{l}\text { Eff. length } \\
\quad(\mathrm{mm})\end{array}$ & $\begin{array}{l}\text { Eff. diameter } \\
(\mathrm{mm})\end{array}$ & $\begin{array}{c}\text { Response } \\
\text { (nC/Gy) }\end{array}$ & $\begin{array}{l}\text { Density of material } \\
\qquad\left(\mathrm{g} / \mathrm{cm}^{3}\right)\end{array}$ \\
\hline \multirow[t]{2}{*}{ Scanditronix } & Filtered photon field diode & PFD & $\sim 0$ & 0.5 & 2 & 35 & 2.3 \\
\hline & Unfiltered stereotactic field diode & SFD & $\sim 0$ & 0.5 & 0.6 & 6 & 2.3 \\
\hline PTW & Diamond detector & 60003 & & 0.25 & 4.5 & & 3.5 \\
\hline
\end{tabular}

The frequency of this check should be determined by the clinic but should be no less frequent than the calibration of the local secondary standard.

II.A.1.d. Stem effect. Irradiating a portion of the ionization chamber stem (cable or holder) can induce leakage current, and this will perturb the collected charge. Because the signal from a small-volume ionization chamber is much weaker than in a large-volume ion chamber, the relative stem effect may be more significant. The stem effect for small ionization chambers has not been well studied in the current literature. The impact of this effect on IMRT dose measurements, especially in low-dose regions, has not been quantified.

II.A.l.e. Acceptance testing. New ionization chambers need to be tested for appropriate performance. These tests should be performed before a calibration is obtained for the chamber. The following suggested tests are adapted from Humphries and Purdy: $:^{31}$

(1) Perpendicular radiographs are taken to verify that the chamber is mechanically sound, comparing with mechanical design drafts if possible.

(2) Leakage current should be measured to ensure that the chamber has a sufficiently low background and that any guard electrodes are performing properly. A bias voltage of 300-350 V should yield at most $(1-5) \times 10^{-14}$ A after a few minutes from turn on or irradiation.

(3) Stem effect should be quantified by irradiating the ionization chamber at orientations that include and exclude the guarded portions of the chamber. ${ }^{2}$ If the guard electrodes are properly working, there should be a negligible difference in readings.

(4) Microphonics (currents generated by mechanically flexing the cables) should be tested in the chamber as well as the cables and connectors that will be used with the ionization chamber. The system should be placed under stress that is similar to any scanning motion and flexing that can exist during phantom measurements. Triaxial cables with a Teflon dielectric are preferred.

(5) Radiation equilibration time should be measured. A properly working ionization chamber should provide reproducible measurements after at most two readings of approximately $200 \mathrm{cGy}$ each.

(6) Atmospheric communication should be tested by checking that the chamber obeys ideal gas law scaling of sensitivity by mildly changing the temperature of the system. Severe temperature changes can cause condensation inside the chamber or possible thermal damage (cracking of plastics).

(7) Polarity effects should be determined to be small
$(<1 \%)$ for a properly working ionization chamber with sound cables. For example, AAPM Task Group 51 states that the polarity effect for a $6 \mathrm{MV}$ beam is unlikely to exceed $0.3 \%$.

(8) Collection efficiency should be measured. The recombination factors of the ionization chamber in the intended radiation fields for calibration should be small (definitely $<1.02$ and typically $<1.015)$ so that accurate corrections can be made.

(9) Orientation dependence needs to be determined and checked against specifications given by the manufacturer. Orientation sensitivity can limit the utility of the ionization chamber.

\section{II.A.2. Solid state dosimeters}

$p$-type semiconductor diode detectors have some attractive characteristics for megavoltage photon beam dosimetry, especially for small-field measurements. Table II lists some of the commercially available megavoltage diodes and their main characteristics. Diode detectors usually have very small active volumes and high sensitivity to radiation. Comparing Table II with Table I reveals that not only is the active volume of diodes much smaller than the smallest ionization chamber but also the sensitivity of these diodes can be 20 100 times greater.

The relatively high atomic number of silicon in the diode detector will lead to a greater sensitivity to low-energy photons. Therefore, diode detectors are usually used for smallfield dose distribution measurements where there are relatively few low-energy photons. ${ }^{32-34}$ Sometimes, low-energy filters, also known as shields, are included in the diode construction to minimize the low-energy sensitivity. These are termed energy-compensated diode detectors. ${ }^{35}$ While these diodes have been shown to have good energy response for on-axis measurements, their use has not been verified for off-axis radiation field measurements.

Diode detector response also exhibits orientation dependence. For beams entering perpendicular to the diode axis, the magnitude of this directional dependence is about $3 \% .^{32}$ However, when the beam is oriented near the diode axis, the diode sensitivity can vary by as much as $15 \%$ (Technical Data Sheet, photon field diode detector, IBA Dosimetry). In addition to the directional dependence, some reports also indicated long-term irreversible radiation damage that changes the sensitivity of diodes over time. ${ }^{36-40}$ Therefore, it is rec- 
TABLE III. Summary of some commercially available electrometers.

\begin{tabular}{|c|c|c|c|c|c|c|c|c|c|}
\hline Vendors & Description & Model & $\begin{array}{c}\text { Accuracy } \\
(\%)\end{array}$ & $\begin{array}{c}\text { Linearity } \\
(\%)\end{array}$ & $\begin{array}{l}\text { Stability } \\
(\% / y r)\end{array}$ & Display & $\begin{array}{l}\text { Minimum charge } \\
(\mathrm{pC})\end{array}$ & $\begin{array}{c}\text { Minimum current } \\
(\mathrm{pA})\end{array}$ & $\begin{array}{l}\text { Leakage } \\
\left(10^{-15} \mathrm{~A}\right)\end{array}$ \\
\hline \multirow[t]{2}{*}{ CNMC } & Reference electrometer & 206 & 0.2 & 0.05 & 0.1 & 4 1/2-digit & 0.0001 & 0.001 & $<3$ \\
\hline & Modified Keithley 602 & K602 & 0.1 & 0.10 & & 4 1/2-digit & 0.01 & 0.001 & $<5$ \\
\hline PTW & UNIDOS & $\mathrm{T} 10005$ & 0.5 & 0.50 & 0.5 & 4 1/2-digit & 0.01 & 0.001 & - \\
\hline Wellhofer & Reference electrometer & DOSE 1 & 0.2 & 0.25 & 0.1 & digital & 0.1 & 0.1 & $<10($ typical $\sim 1)$ \\
\hline
\end{tabular}

ommended that diodes be used only for relative dose measurements and that care should be taken when selecting diode orientation.

In contrast to silicon diode detectors, diamond detectors are almost soft-tissue equivalent in terms of atomic composition (although they have a physical density much greater than water at $3.5 \mathrm{~g} \mathrm{~cm}^{-3}$ ), exhibit a small directional dependence, have good mechanical stability, and high radiation hardness $^{21,35,41-43}$ (lack of radiation sensitivity variation due to irradiation). Similar to silicon diodes, diamond detectors also exhibit high radiation sensitivity. Bucciolini et al. ${ }^{21}$ demonstrated that diamond detectors can produce clinically identical results as $p$-type silicon diodes for small treatment fields. However, dose-rate dependence may affect the ability of using diamond detectors for scanning or for measurements of IMRT dose distributions. ${ }^{21}$

Recently, chemical vapor deposition (CVD) diodes have been investigated for radiation therapy dosimetry. ${ }^{44-54} \mathrm{~A} \mathrm{Eu}$ ropean Integrated Project (IP) called MAESTRO (Methods and Advanced Equipment for Simulation and Treatment in Radiation Oncology, http://www.maestro-research.org/ index.htm) is developing a range of new tools for providing dose measurements and the group is concentrating on IMRT dose validation. One of their development efforts is to examine diamond detectors as quantitative radiation dosimeters.

Another type of small-field dosimeter that has been used in IMRT is the thermoluminescence dosimeter (TLD). ${ }^{55-68}$ TLD dosimetry has been used since the 1970s to quantify megavoltage $\mathrm{x}$-ray dosimetry. ${ }^{69-73}$ A TLD is an integrating dosimeter, which is usually small in size, often in the form of a cubical or cylindrical chip, and has a nearly tissueequivalent atomic composition $(Z=8.1)$ and a typical physical density of $2.6 \mathrm{~g} \mathrm{~cm}^{-3}$. Typical TLDs can exhibit nonlinear integrated dose response, which must be evaluated before use, ${ }^{74}$ along with some energy response variations. The energy response variation is sufficiently large to require that the sensitivity calibration should be conducted using the same megavoltage beam energy as the experiment. TLD implementation is labor intensive, so it is typically employed where ionization chamber measurements are impractical, for example, in multiple anthropomorphic phantom dose measurements. To achieve dose measurement precision on the order of $2 \%-3 \%$, a TLD implementation program requires a rigorous annealing and response measurement protocol, and routine QA of the TLD reader and annealing oven temperature control. ${ }^{75}$

\section{II.A.3. Electrometer and cable performance}

The basic requirements for electrometers are (1) accuracy, (2) linearity, (3) stability, (4) sensitivity, (5) high impedance, and (6) low leakage. Table III lists some of the performance data of modern electrometers according to vendors' literature. These electrometers have much lower leakage currents than most of the older models. The leakage for ionization chambers shown in Table I is comparable to the electrometers' leakage.

With respect to small ionization chambers used for IMRT, the performance of the read-out electrometer becomes more important as the ionization chamber volume is reduced. The fraction of integrated charge due to cable and electrometer leakage is inversely proportional to the charge collected by the chamber and is proportional to the amount of time required for the measurement. In IMRT dose measurements, the dose integration time is often significantly longer than for conventional measurements. ${ }^{76-81}$ A large fraction of the delivered dose is due to collimator and linear accelerator head leakage, so the dose needs to be measured for all beams, even those radiation beams that do not directly intercept the ionization chamber (for example, for serial tomotherapy indices where the chamber position is far from the beam portals).

The leakage of the chamber-cable-electrometer system should be checked for each measurement session using the commonly accepted leakage specification published by Humphries and Purdy. ${ }^{31}$ It states that "Excellent, average, and bad electrometer zero drifts are $10^{-15} \mathrm{~A}, 10^{-14} \mathrm{~A}$, and $10^{-13}$ A, respectively." The contribution of background leakage to the integrated charge can be approximated as a function of ionization chamber volume, integrated dose, and integration time. Assuming that the leakage current linearly affects the resulting measured charge, the relative error in integrated charge measurements is

$$
E=\frac{\left(C+I_{l} t\right)}{C},
$$

where $E$ is the relative measurement error, $C$ is the charge due to ionizations in the chamber, $I_{l}$ is the leakage current, and $t$ is the ionization integration time.

Figure 1 shows some examples using Eq. (1) applied to an integrated dose of $200 \mathrm{cGy}$ for typical ionization chamber volumes. These data suggest that care should be taken when using ionization chambers with relatively small volumes if 

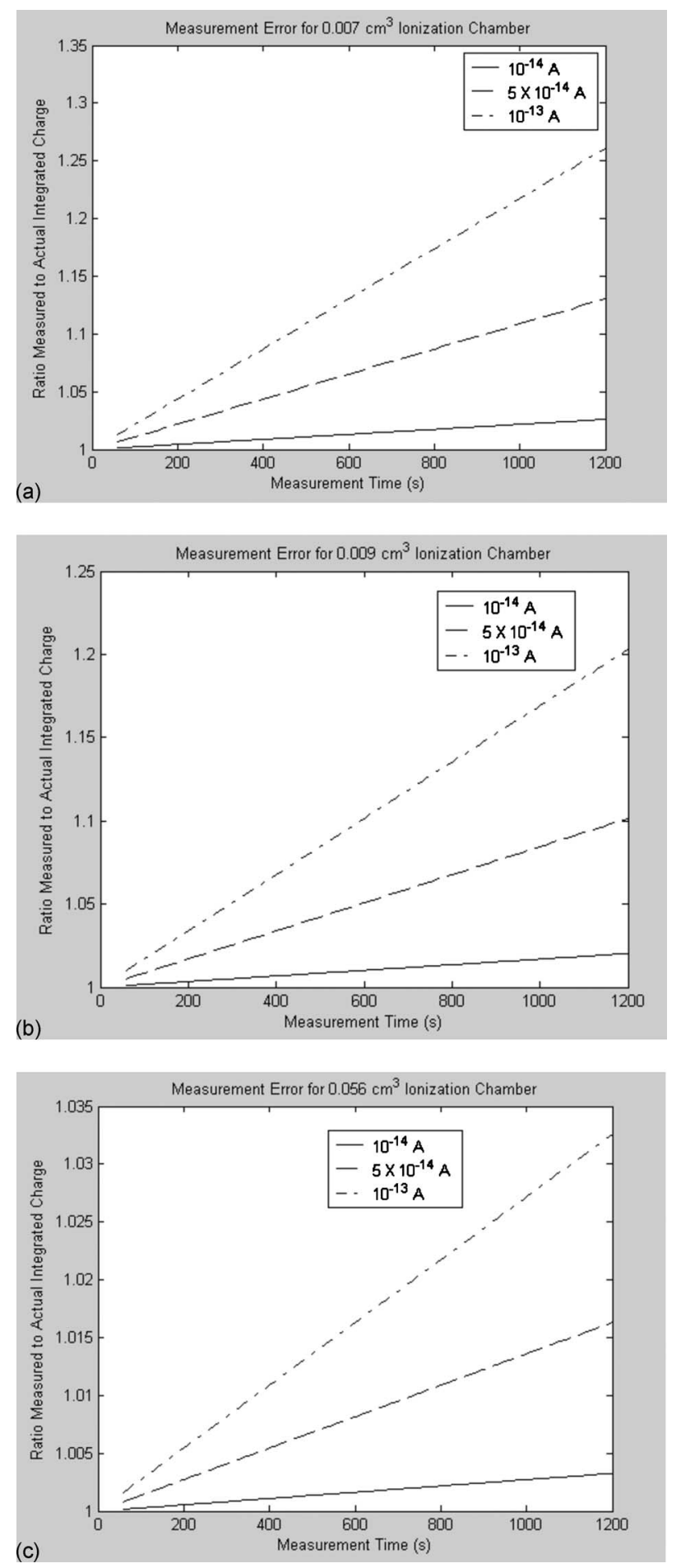

FIG. 1. Examples of fractional measured ionization collection errors due to system leakage current for a $200 \mathrm{cGy}$ IMRT dose distribution measurement for a variety of ionization chamber volumes and system leakage currents. (a) Measurement error for a $0.007 \mathrm{~cm}^{3}$ volume ionization chamber. (b) Measurement error for a $0.009 \mathrm{~cm}^{3}$ volume ionization chamber. (c) Measurement error for a $0.056 \mathrm{~cm}^{3}$ volume ionization chamber.

the measurements are expected to require several minutes of ionization collection. For example, if an uncorrected IMRT measurement of $200 \mathrm{cGy}$ requires $10 \mathrm{~min}$ to acquire, using a $0.009 \mathrm{~cm}^{3}$ ionization chamber, leakages of $10^{-14}, 5 \times 10^{-14}$, and $10^{-13}$ A will yield $1 \%, 5 \%$, and $10 \%$ dose measurement errors.
Physicists should also assess the performance of electrometers with automatic leakage correction. The correction feature may need to be turned off for low-dose IMRT measurements so that the reported collected charge is not incorrectly reset during a measurement.

\section{II.A.4. Applications to IMRT}

II.A.4.a. Selection of radiation detectors for IMRT measurements. For point-dose measurements, an ionization chamber built from tissue- or air-equivalent materials is recommended to minimize detector over-response to variations in the photon spectrum as a function of measurement position. Because of the steep dose gradients and relatively large number of small-field size segments used in IMRT plans, it is essential that the ionization chamber have a spatial resolution of sufficient magnitude to avoid dose measurement errors. When comparing calculations against measurements, the calculated dose can be determined either by point sampling in the calculated dose distribution or extracting the mean ionization chamber volume dose. This is typically conducted by contouring the active chamber volume in the treatment plan and using dose-volume statistics to determine the average dose value. The volume-averaging process is recommended unless it is not possible or impractical. If the volumeaveraging process is used, the ionization chamber volume can be larger and this can reduce the sensitivity of spatial positioning error on the measured dose.

The doses being delivered to critical structures can also be verified using an ionization chamber. As with target volumes, care should be taken to assure that the dose is relatively homogeneous across the ionization chamber and that measured dose be compared against the volume-average of the calculated dose.

II.A.4.b. Preparing for IMRT measurements. The preparation for ionization chamber measurements should include allowing the ionization chamber and phantom to reach equilibrium temperatures (e.g., the treatment room temperature) and orienting and localizing the chamber and phantom accurately with respect to the linear accelerator. Better than $1 \mathrm{~mm}$ positional agreement with respect to the isocenter between the measurement system and the calculated system should be possible.

II.A.4.c. Cross-calibration of detectors prior to IMRT measurements. Clinics typically use a Farmer-type ionization chamber as the local secondary standard. A local crosscalibration procedure to assess the IMRT ionization chamber response should be established prior to performing IMRT measurements. For example, Dong et al. ${ }^{14}$ described a crosscalibration procedure that used lateral parallel-opposed 10 $\times 10 \mathrm{~cm}^{2}$ fields to monitor the linear accelerator output variation at the time of measurement. Another approach is to determine an ionization chamber and electrometer sensitivity factor using a calibrated radiation beam and then to use the measured sensitivity factor for subsequent IMRT QA procedures. This approach will provide dose measurements that are sensitive to the daily linear accelerator output fluctuations. This procedure should be conducted no less often than the calibration of the local secondary standard. 
II.A.4.d. Output factor measurements. Dose measurement errors can occur if small-field output measurements are conducted with large-volume ionization chambers. Smallvolume ionization chamber measurements without volumeaveraging corrections should be used for field sizes between $2 \times 2$ and $5 \times 5 \mathrm{~cm}^{2}$ such that the field sizes are $1.5 \mathrm{~cm}$ wider than the effective length of the ionization chamber. Correction techniques, such as the deconvolution method, have been used to obtain accurate relative output measurements using small-volume ionization chamber measurements, ${ }^{22,82-84}$ but they are not in widespread use. For larger field sizes, standard-volume ion chambers can be used. Alternatively, radiographic or radiochromic film or diodes can be used to determine the output factors for very smallfield sizes. ${ }^{85-89}$

II.A.4.e. Profile and central axis depth-dose measurements. IMRT treatment planning systems require profile and depth-dose measurements as input for modeling and verification. Photon beam profiles have steep dose gradients and, consequently, the spatial resolution of the measuring detector can profoundly affect the measured profile accuracy. Even small-volume ionization chambers will artificially broaden the measured penumbra shape due to volume averaging. ${ }^{16,20}$ Using the ionization-chamber measured penumbra in the treatment planning system modeling process may cause errors in subsequent IMRT dose calculations and MLC leaf sequences. ${ }^{90,91}$ High-spatial resolution measurement systems (effective resolution $<1 \mathrm{~mm}$ ), such as radiographic and radiochromic film coupled with quantitative film densitometers, or scanning diodes, will provide sufficiently accurate measurements of the penumbra shape for treatment planning system dose modeling.

Aside from radiochromic film, the high-spatial resolution detector systems have non-water-equivalent energy response. The measurements using these systems will be more accurate if they are confined to relative measurements where the beam energy spectrum does not vary significantly within the measured distribution. The photon energy spectrum changes sufficiently with respect to depth for central axis depth doses, for example, so that the energy response of the non-waterequivalent detectors can cause measurement errors. There are also ionization chambers that have non-water-equivalent response. An example is the steel-electrode PinPoint ionization chamber (Table I) dose response reported by Martens et al. ${ }^{20}$ While this ionization chamber provided excellent profile measurements, the steel electrode caused energy response variations that made the chamber unsuitable for output factor measurements or depth-dose measurements. Recently, Agostinelli et $a{ }^{92}{ }^{92}$ reported the energy response for a new version of the PinPoint ionization chamber with an aluminum electrode. When a polarity correction measurement was used, the ionization chamber response varied less than $0.5 \%$ for field sizes from $2 \times 2$ to $40 \times 40 \mathrm{~cm}^{2}$. It is incumbent on the physicist to conduct the sensitometric measurements at depths and field geometries that most closely match the profile measurement conditions so that differences in the photon spectrum yield insignificant sensitivity differences.

\section{II.A.5. Summary of recommendations}

(1) Ionization chambers

(a) Ionization chambers should be used:

(i) In homogeneous dose regions

(ii) To verify monitor unit outputs

(iii) To verify critical structure doses.

(b) Ionization chambers should not be used:

(i) To measure beam profiles that will be employed to model the IMRT beam penumbra.

(ii) When the leakage current will yield an integrated charge of $>5 \%$ of the expected radiation-induced charge. Leakage current corrections should be applied if the expected uncorrected error is $>2 \%$.

(c) Chamber selection

(i) A cylindrical ionization chamber should be used.

(ii) The size of the ionization chamber should be appropriate for the task

(1) For measuring output factors: The radiation field should be $1.5 \mathrm{~cm}$ wider than the effective length of the ionization chamber.

(2) For IMRT dose measurements: The ionization chamber size should be small enough to limit the dose heterogeneity across the chamber active volume to $10 \%$ and $5 \%$ if the measurements are being compared against volumeaveraged and point doses, respectively.

(iii) The ionization chamber electrode should be fabricated out of low- $Z$ materials (e.g., aluminum). When high- $Z$ electrodes are used, the chamber should be cross-calibrated in conditions that minimize photon spectral differences (e.g., the same depth and minimizing field size differences).

(d) Measurement protocols

(i) When ionization chambers are used to validate single-point IMRT absolute doses:

(1) The ionization chamber should be placed in a region of the dose distribution where the expected dose heterogeneity is less than $10 \%$ or $5 \%$ across the ionization chamber and the expected dose heterogeneity is less than $10 \%$ within $2 \mathrm{~mm}$ from the intended ionization chamber position, if the measure- 
ments are being compared against volume-averaged or point doses, respectively.

(2) While point-dose comparisons are possible with all treatment planning systems, the measured dose should be compared to the calculated doses that have been averaged throughout the active ionization chamber active volume. This is typically done by computing the dose using a CT scan with the ionization chamber in place, contouring the ionization chamber volume, and querying the treatment planning system dose statistics for the mean dose. This system can also be used to identify the expected dose heterogeneity.

(2) Diode detectors (single-point detectors)

(a) Diode detectors should be used:

(i) For measuring relative dose distributions, particularly for measuring MLC penumbras used in beam modeling in a treatment planning system

(ii) For providing dose measurement points supplemental to ionization chamber measurements

(b) Diode detectors should not be used:

(i) To measure absolute doses

(ii) As the sole measurement device for measuring beam profiles that will be employed to model the IMRT beam penumbra. This is due to the potential for over-response in the low-dose regions outside the field boundaries. Other detectors with little energy response variations should be compared to diode-measured profiles to assure that the diode profile provides accurate out-of-field results.

(c) Diode selection

(i) Use unshielded diode detectors

(ii) Diode detectors designed for in vivo dosimetry should not be used for in-phantom measurements.

(d) Measurement protocols

(i) Diode response varies with orientation, so the relative orientation of the diode to the radiation beam should be carefully considered.

(3) Thermoluminescent dosimeters (chips)

(a) TLD detectors should be used: (i) When the phantom geometry will not allow ionization chamber measurements.

(ii) When multiple simultaneous point measurements are desired.

(b) TLD detectors should not be used:

(i) To measure absolute doses if the overall measurement precision needs to be better than $3 \%$

(c) TLD selection

(i) Low-atomic number TLDs (e.g., LiF) should be used.

(d) Measurement protocols

(i) A strict annealing and calibration protocol should be adopted that provides relative response factors for individual chips.

(ii) Care should be taken to assure that the user accurately knows the TLD positions with respect to the linear accelerator.

\section{II.B. Two-dimensional dosimetry}

While point dosimetry allows for absolute IMRT dose distribution validation at individual points, thorough validation and quality assessment of IMRT dose distributions requires higher dimensional measurements. The current commercial options for two-dimensional (2D) dosimetry are radiographic film, radiochromic film, computed radiography, diode arrays, and ionization chamber arrays.

\section{II.B.1. Film}

II.B.1.a. Silver halide film. This section describes the relative 2D radiographic film dosimetry techniques for IMRT validation and quality assurance. A report from the American Association of Physicists in Medicine (AAPM) Task Group 69 on radiographic film for megavoltage beam dosimetry comprehensively addressed all aspects of radiographic film dosimetry and we refer the reader to this document for a more detailed discussion. ${ }^{93}$

Although radiographic film has been demonstrated to be capable of effectively validating 2D IMRT dose distributions in phantoms, there are many important considerations and potential pitfalls in using this technique. These include variations in film sensitivity caused by film batch, processor conditions, variations in photon beam spectrum throughout the measured dose distribution, and densitometry artifacts. Of these variables, the one that has the greatest potential for causing dosimetry errors is the variation in optical density (OD) with film processor developer temperature (TG 69 and Bogucki et al. ${ }^{94}$ ).

When considering the trade-off between experimental complexity and labor and the information obtained, radiographic film provides an attractive measurement technique. Radiographic film dosimetry is convenient to employ because high quality, automated film-processing equipment is 
still available in most clinical settings. In general, when matched with the appropriate densitometer, film is an excellent tool for 2D dose mapping due to its extremely highspatial resolution with grain sizes typically having dimensions on the order of microns.

II.B.1.b. Radiochromic film. Unlike radiographic film, radiochromic film is nearly tissue-equivalent and does not require a processor for generating the optical density response to ionizing radiation. An AAPM Task Group (TG 55) (Ref. 95) reported on the use of radiochromic film for dosimetry measurements. Until recently, most of the experience using radiochromic film was based on Gafchromic MD-55 and HS, which were relatively insensitive to radiation. ${ }^{86,87,96-103}$ The dose sensitivity was too low for the film to be practical for routine clinical IMRT measurements. Recently, a highsensitivity radiochromic film (Gafchromic EBT) was introduced that has OD sensitivity similar to the radiographic Kodak EDR-2 film. ${ }^{104-112}$ The current fabrication process for EBT film places the radiation sensitive layer within the substrate in such a way that the measured OD can be a strong function of the film orientation. ${ }^{113}$ Laser densitometers are not recommended for use with the film because the crystalline structure for this type of film has changed from that of previous films, causing polarization artifacts and lead to a steep orientation dependence of OD response. ${ }^{114}$ Initial investigation of fluorescent lamp-based flatbed scanners with the new film yielded promising results. ${ }^{84,115}$ Published works indicate that there are three significant artifacts that can limit the accuracy of Gafchromic EBT read out on flatbed scanners. ${ }^{113,114,116}$ The first effect is a film rotation effect that depends on the orientation of the film on a flatbed scanner bed. The second involves scanner uniformity, and the third depends on the temperature of the scanner bed while scanning. In spite of these limitations, radiochromic film is the only available alternative for high-spatial resolution 2D dose measurements for clinics that have not retained or installed radiographic film processors.

Potential users of EBT film should be aware that clinical implementation of radiochromic film poses additional challenges that differ from radiographic film. The current formulation of EBT film utilizes a hydroscopic emulsion that causes variation in sensitivity immediately after the films are cut, typically done to place them in a phantom or to prepare for a sensitometric measurement. Some cutting techniques cause more shearing that leads to greater film edge artifacts, so the user should develop a cutting technique that minimizes shearing.

At typical dose levels, the pixel-to-pixel measurement noise is greater than other dosimetry techniques in radiation therapy. For data used in the measurement of beam profiles or output factors, the dose to the film can be increased such that the noise is relatively small. When radiochromic film is used to measure clinical IMRT dose distributions in low-dose regions, or to characterize low-dose features such as penumbrae tails, the pixel-to-pixel noise may limit the measurement precision. Methods such as pixel averaging can be used to reduce the noise, but caution should be taken to assure that these methods do not introduce errors in the measured doses.
As with other film methods, all films within an experiment should be consistently handled, for example, storage conditions, time between irradiation and scanning, and orientation in the scanner. The sensitivity to humidity has not yet been quantified and may be a significant cause of optical density variations.

The manufacturer recently developed a new formulation and markets it as EBT-2. The manufacturer claims that EBT-2 has improved properties over the previous film, but the user should verify its dosimetric properties prior to use.

II.B.1.c. Densitometry. After processing, films need to be scanned to measure their response. Transmission-based optical scanners are required for good quantitative results. When properly calibrated and characterized, commercially available 2D optical densitometers allow for the study of the steep dose gradients found in IMRT dose distributions. For the purposes of this report, we divide the scanner designs into two classes. Confocal point-source scanners translate a point source over the film, typically by translating a collimated beam of light in concert with a confocal detector (single source focused to a detector such that measurements are made point-by-point). This type of scanner typically has the poorest spatial resolution with beam spot sizes between 0.25 and $0.8 \mathrm{~mm}$ in diameter. Sample spacing for these devices can be typically adjusted between a fraction of the spot size and several centimeters. Higher-dimensional scanners employ linear or area arrays of detectors and a light source. Commercially available 2D scanners typically have pixel dimensions that are defined by the light source and detector geometry. These devices have a much higher spatial resolution with pixel dimensions between 0.34 and $0.042 \mathrm{~mm}$. The detectable OD for these devices typically ranges between $0 \mathrm{OD}$ and an upper limit of between 2.5 and 4.0 OD, depending on the light source and detector technology. Measurements should be limited to the OD dynamic range specified by the scanner manufacture to ensure reliable results.

Before using a scanning system for film densitometry, the response, spatial integrity, susceptibility to image artifacts, and quality assurance protocol of the scanner should be checked according to the recommendations of the AAPM TG69. It is important to establish a protocol of operation so that results are measured consistently, for example, assuring that the scanner reaches steady-state operating conditions.

II.B.1.d. Application to IMRT. Radiographic film. Silver halide based radiographic films, often referred to as radiographic films (although all dosimetry films are, in fact, radiographic), have been widely used for validation of the relative dosimetry of IMRT treatment phantom plans. ${ }^{56,65,68,90,106,117-127}$ In principle, such measurements allow for the verification of the appropriate shape and registration of the IMRT dose distribution in a selected twodimensional plane (film plane). Appropriate selection of the orientation and locations of the film planes is important to assure that the measured dose is useful for the desired QA function.

The most important quantity to consider when selecting a type of film for dosimetry is the dependence of OD on the absorbed ionizing radiation dose, often called the sensitomet- 
ric or characteristic curve. There are numerous commercial manufacturers of radiographic films, including Agfa, CEA, DuPont, Fuji, Kodak, and Konica. These companies make a wide range of films for different applications. Currently in the United States, the majority of clinical film dosimetry is performed with two films from a single manufacturer: Kodak XV2 and EDR2 film. These films differ mainly in the shape of their characteristic curves and the speed of the films. In order to measure an IMRT dose distribution without perturbing the clinical delivery process, a film is required to accurately measure doses up to the maximum delivered dose. For dose measurements of individual fields, either Kodak XV2 or EDR2 film is useful. For composite dose measurements of entire IMRT dose distributions, EDR2 film is most appropriate because it is the only film type with a characteristic curve that maintains a useful sensitometric slope up to and beyond $500 \mathrm{cGy} .{ }^{121,127-129} \mathrm{Zhu}$ et al. ${ }^{129}$ showed that the sensitometric curve maintains a slope until $600 \mathrm{cGy}$, but the net OD at that point was 3 , which can cause densitometry artifacts for some scanners. In order to allow the OD of EDR2 film to stabilize, the film should be processed at least $1 \mathrm{~h}$ after irradiation. ${ }^{130}$

Radiochromic film. Because of its nearly tissue-equivalent characteristics and well-matched dose response, radiochromic film can be used to acquire accurate relative dose distribution measurements. ${ }^{95,105,131-134}$ For IMRT commissioning, radiochromic film can be used to acquire beam profiles that will be used for modeling beam penumbra. ${ }^{99,133}$ It can also be used to measure relative output factors for very small fields ${ }^{100,133,135-137}$ and can be used to measure phantombased IMRT dose distributions, for example, dose distribution measurements that will be used to verify the treatment planning system's programming of the linear accelerator delivery parameters ("monitor unit outputs").

Although radiochromic film has a nearly linear OD dependence, accurate dose measurements still require a custom-measured sensitometric curve. ${ }^{99}$ This is typically conducted by cutting up a piece of radiochromic film into relatively small pieces (approximately $3 \times 3 \mathrm{~cm}^{2}$ ) and irradiating each piece to a different dose. Even though EBT film is nearly tissue-equivalent, the calibration beam should be of the same energy as the measurement energy. In order to allow the OD response of the film to stabilize, the film should not be scanned less than $1 \mathrm{~h}$ after irradiation. ${ }^{99}$

II.B.1.e. Summary of recommendations.

(1) Film

(a) Radiographic film

(i) Radiographic film should be used:

(1) For relative IMRT dose distribution measurements

(2) To measure beam profiles that will be employed to model the IMRT beam penumbra

(3) For measuring relative output factors of small fields

(ii) Radiographic film should not be used:
(1) For absolute dose measurements

(2) To verify monitor unit outputs

(iii) Film selection

(1) Two commercial radiographic films are most commonly used, Kodak EDR2 and XV2. The selection of film should be based on the expected maximum dose in the film plane.

(2) XV2 should not be used to measure doses greater than $100 \mathrm{cGy}$.

(3) EDR2 should not be used to measure doses greater than $500 \mathrm{cGy}$.

(iv) Measurement protocols

(1) A sensitometric curve should be measured for each radiographic film experiment.

(2) The sensitometric curve films should be selected from the same batch as the measurement films.

(3) The sensitometric curve films should be processed at the same time as the measurement films.

(4) The recommendations of AAPM Task Group 69 should be observed for radiographic film and densitometry.

(5) Handle film carefully with clean hands or light cotton gloves.

(6) Bending, stretching, or scratching films should be avoided.

(7) For EDR-2, wait at least $1 \mathrm{~h}$ after irradiation before processing.

(b) Radiochromic film

(i) Radiochromic film should be used:

(1) For measuring relative dose distributions

(2) For measuring dose distributions that will be used to model the IMRT beam penumbra

(3) For measuring relative output factors of small fields

(4) When a radiographic film processor is not available

(ii) Radiochromic film should not be used:

(1) For absolute dose measurements

(2) To verify monitor unit outputs

(iii) Film selection

(1) EBT-2 is the only film commercially available with appropriate sensitivity

(iv) Measurement protocols 
(1) A sensitometric curve should be measured for each radiochromic film experiment.

(2) The sensitometric curve films should be selected from the same batch as the measurement films.

(3) The optical density distribution should be measured no sooner than $1 \mathrm{~h}$ after irradiation

(4) The sensitometric curve and measurement films should be irradiated on the same day

(5) The sensitometric curve and measurement films should be scanned on the same day

(6) The orientation of the sensitometric and measurement films during scanning needs to be consistent with respect to the original orientation.

(7) Handle film carefully with clean hands or light cotton gloves.

(8) Bending, stretching, or scratching films should be avoided.

\section{II.B.2. Array detectors}

Array detectors calibrated to yield multiple cumulative readings of absorbed dose across a $2 \mathrm{D}$ plane represent a recent and popular new addition to the tools available for routine clinical IMRT QA. ${ }^{106,138-141}$ They offer the potential for increased efficiency because, after cross-calibration, they can be used to provide a large number of dose measurements in a single irradiation (per beam), with the results available immediately after delivery. The immediacy of the results represents an attractive feature for IMRT QA as it facilitates efficient diagnosis of common sources of error and estimation of their magnitude. Examples include errors in leaf calibration and inaccurate penumbral and small-field output factor modeling by the treatment planning system. Correlating measurement discrepancies that lie along the trajectory of a leaf pair can be used to diagnose leaf-positioning errors. Existing array detectors have low spatial resolution (typically $>7 \mathrm{~mm}$ ), which limits their role to routine QA of a precommissioned IMRT technique. Initial commissioning should be performed with a higher resolution system (e.g., film) to enable more thorough evaluation of the fine dose distribution structure. A further limitation to array detectors is that although each beam can be independently verified, no knowledge is obtained about the composite 3D dose distribution, resulting from the superposition of all beams. If errors are determined in individual beams, the accumulation of all errors in the 3D distribution is unknown. Significant discrepancies at this stage can be investigated with planar measurements in 3D phantoms (Sec. II B 1). Despite these limitations, 2D planar detector arrays have achieved widespread clinical acceptance due to their convenience and efficiency.

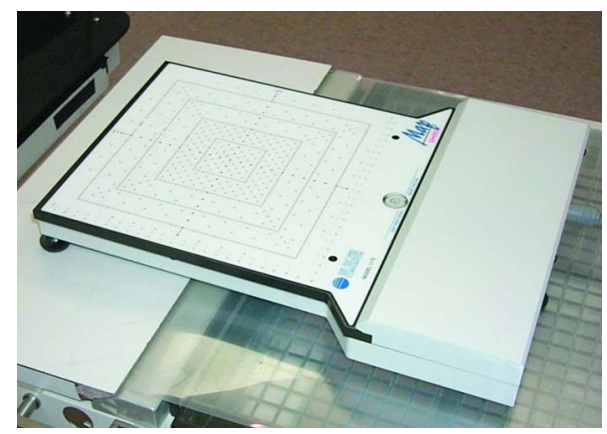

FIG. 2. Example of a matrix detector.

II.B.2.a. Description. Until recently, the only commercially available two-dimensional diode array detector utilized $n$-type diode technology. ${ }^{138,140,141}$ This device is called the Mapcheck (Sun Nuclear, Melbourne, FL) (Fig. 2). The Mapcheck contains an array of 445 variably spaced diodes over an area of $22 \times 22 \mathrm{~cm}^{2}$. The diode spacings are 7.07 and $14.14 \mathrm{~mm}$ in the central $10 \times 10 \mathrm{~cm}^{2}$ and the outer regions, respectively. The diode plane has an effective build-up depth of $2 \mathrm{~cm}$ and a backscattering thickness of $2.3 \mathrm{~cm}$. The physical cross section of each diode is $0.8 \mathrm{~mm}^{2}$. The diode response is linear with dose up to saturation at $\sim 2.8 \mathrm{~Gy}$. A $2 \%$ sensitivity variation has been observed when the instantaneous dose rate is varied by a factor of $3 .{ }^{140}$ Uncertainties arising from these variations can be minimized by standardizing the SSD and repetition rates for IMRT QA. ${ }^{138,140}$

The Mapcheck is calibrated through a straightforward process provided by the manufacturer. A relative detector calibration is conducted first using a series of irradiations of a static field. The detector is rotated or translated between irradiations in a manufacturer-defined sequence. This identifies the relative calibration of each detector. The second step calibrates the Mapcheck device for absolute dosimetry by irradiating the device to a known dose at the central axis. The relative calibration is highly stable, and publications indicate that it is stable for 6 months. ${ }^{106,138,140}$ The system calibration varies with temperature at $\sim 0.5 \% /{ }^{\circ} \mathrm{C} .{ }^{140}$

Ionization chamber array detectors are also under development in academic and commercial institutions. ${ }^{142-144}$ Recently, Poppe et al. ${ }^{145,146}$ reported on two commercial ionization chamber arrays. These have been designed and fabricated by PTW-Freiburg. Version 1 and the model seven 29 cover an area of $27 \times 27 \mathrm{~cm}^{2}$. Version 1 has 256 detectors $(16 \times 16)$ and model seven29 has $729(27 \times 27)$ ionization chambers, both arrayed in a square pattern. Version 1 utilizes $8 \times 8 \mathrm{~mm}^{2}$ cross-sectional chambers with 8 $\mathrm{mm}$ water-equivalent material between adjacent chambers to isolate each chamber relative to secondary electron flux perturbations from neighboring chambers. The model seven 29 utilizes $5 \times 5 \mathrm{~mm}^{2}$ cross-sectional chambers with $5 \mathrm{~mm}$ water-equivalent material between adjacent chambers. The chambers for both version 1 and model seven 29 have $5 \mathrm{~mm}$ air-filled height. The short- and long-term (4 months) reproducibility was found to be $0.2 \%$ and $1 \%$, respectively. The linearity was excellent, being less than $0.4 \%$ from doses of 2 
to $500 \mathrm{MU}$. The measured output factors showed excellent agreement with conventional ionization chamber measurements. ${ }^{145,146}$ Both arrays responded as expected with respect to spatial resolution.

II.B.2.b. Application to IMRT. The Mapcheck device is used to verify the absolute and relative dose distribution on a beam-by-beam basis. The device is leveled on the couch and irradiated at normal incidence with the gantry oriented with the central axis pointing downward. Typically, additional build-up material is placed on the device so that the effective measurement plane is $5 \mathrm{~cm}$ or deeper. For large IMRT fields, it may be necessary to reduce the SSD to maintain the field within the $22 \mathrm{~cm}^{2}$ active area with an equivalent reduction in the SSD used in the phantom treatment plan or multiple measurements may be required with the device at different positions. Software interpolation of the measured dose points generates a $2 \mathrm{D}$ contour map of IMRT dose distribution at the measurement depth. The user can then import the calculated dose distribution corresponding to the same beam and depth for comparison and analysis.

The $2 \mathrm{D}$ ionization chamber arrays have detector spacing of $1 \times 1 \mathrm{~cm}^{2}$, so they may not provide sufficiently highspatial resolution to provide isodose distributions, but each detector point may provide a quantitative dose measurement. Depending on the dose analysis tool employed, even with such a relatively coarse spacing, the detector array may provide a quantitative method for evaluating IMRT dose distributions. Further investigation is warranted to determine the limitations of these arrays for IMRT QA.

II.B.2.c. Recommendations for use.

(1) Useful for efficient routine QA of a precommissioned IMRT technique. Initial commissioning should be performed with a system with higher spatial resolution (e.g., film).

(2) For calibration and all measurements with the device, the linear accelerator dose repetition rate should be the same as for the clinical treatment.

(3) The device calibration should be checked monthly, or as specified by the manufacturer or published literature.

(4) Careful consideration should be given to the development of pass/fail acceptance criteria for the evaluation of the results from an array detector. For example, AAPM Task Group 119 (Ref. 17) demonstrated pass rates of $>90 \%$ of the evaluated points when using $3 \mathrm{~mm} / 3 \%$ distance-to-agreement (DTA) and dose-difference criteria, respectively, when reporting institution's planar diode detector measurement QA results. Each physicist should determine acceptance criteria that are appropriate for the treatment site, the treatment objectives, and the clinic's policies.

\section{II.B.3. Computed radiography}

Computed radiography (CR) has been available for more than 20 years, ${ }^{147}$ but is gaining interest in radiation oncology as a dosimeter because of the removal of film processors from radiation oncology departments. CR uses a storage phosphor to temporarily record a radiographic image. The storage phosphors use barium, which causes the phosphors to over-respond to low-energy photons. Also, while the systems are used for radiology, their use as quantitative dosimeters still has the following challenges: Response stability, reader optical scatter, sensitivity to room light exposure, and directional response variations. ${ }^{148} \mathrm{CR}$ techniques have been successfully used for megavoltage beam relative dosimetry by employing low-energy filters, but care should be taken if CR is used for IMRT QA because the photon spectrum varies widely across IMRT fields. CR techniques have been successfully used for relative dosimetry of single and composite (axial plane) megavoltage beam IMRT using low-energy filters for field sizes less than $15 \times 15 \mathrm{~cm}^{2}{ }^{148}$

\section{PHANTOMS}

\section{III.A. Phantom types}

\section{III.A.1. Phantom selection for IMRT}

Verification processes for IMRT vary significantly in their phantom requirements, with the appropriate phantom determined by the purpose of the measurement. Phantoms are typically constructed using either water or water-equivalent plastic. ${ }^{65,68,149-152}$ Open water phantoms can be used when the beam is perpendicular to the phantom surface, and where great flexibility in detector positioning is desired. With the proper procedures and design, water-equivalent plastic phantoms can support multiple detectors, radiographic film, and rapid and efficient setup reproducibility. Such phantoms can also include the substitution or addition of heterogeneous materials. ${ }^{65,150}$ To conduct an overall evaluation of an IMRT delivery system, anthropomorphic phantoms are useful in conjunction with other phantoms.

\section{III.A.2. Geometric phantoms}

Simple geometric phantoms that can accommodate ionization chambers and film are used for measuring singlepoint and planar doses. ${ }^{65,150}$ Cubic phantoms, comprised of slabs, are easy and accurate to set up and allow for measurements at multiple depths. The slabs can be water equivalent or built with materials having relative electron densities representing specific anatomical tissues. Setup accuracy is improved if the phantom is scribed with setup lines whose positions are accurately known with respect to the dosimeters. The use of fiducial marks on the film should be considered for registration of the film with respect to the phantom. For example, a NOMOS phantom was modified to accommodate TLDs and multiple ionization chamber positions by using different spacers of water-equivalent material. ${ }^{65}$ The use of the phantom was also extended from a head-and-neck geometry to a prostate geometry by construction of an outer shell to fit over the rectangular phantom. Rectangular phantoms are useful for measuring single field or composite dose distributions.

Cylindrical phantoms have a convenient geometry for coplanar composite IMRT delivery verification, while allowing for multiple ionization chamber positions. ${ }^{153}$ A novel cylin- 
drical phantom that places radiographic film in a spiral slot and an ionization chamber at the phantom center was developed for tomotherapy verification. ${ }^{68,151}$ The advantage of this phantom is that it samples the volumetric dose distribution with a single film, although film dosimetry analysis software vendors have not provided software that interpolates the calculated dose distributions to the coordinates of the measured film. Another cylindrical water-equivalent phantom is provided by Tomotherapy, Inc., for the quality assurance of Tomotherapy treatments. ${ }^{154}$ There are also slab phantoms that are machined to accept commercial ionization chambers.

\section{III.A.3. Anthropomorphic phantoms}

Anthropomorphic phantoms are fabricated in the shape of a human and, consequently, they can be more difficult or at least more time consuming to accurately set up and align with respect to the linear accelerator. The preparation and accurate placement of film can be more difficult than with geometric phantoms. The preparation of films involves cutting film to match the shape of the external phantom shape and sealing the phantom around the film with light-tight tape if light-sensitive films are used. In spite of the added difficulty, anthropomorphic phantoms have been effectively used for limited measurements to evaluate the process of patient treatment planning and delivery and to identify treatment planning or dose delivery problems that are not evident in simple homogeneous geometric phantoms. ${ }^{59,155-157}$ The phantom setup typically parallels a human simulation and irradiation. For example, a CT simulation of the phantom should be conducted using radiopaque and visible fiducial markers, and when possible, the phantom position should be independently verified, for example using an electronic portal imaging device (EPID) or film at the treatment unit before delivery.

While anthropomorphic phantoms are good for assessing the overall IMRT planning and delivery process, many commercial phantoms are composed of thick transverse slices, which limit the flexibility in film and point-dosimeter placement. Another problem is that causes of dose distribution discrepancies are difficult to isolate using an anthropomorphic phantom. Therefore, additional measurements using geometric phantoms may also be required to aid in the interpretation of any discrepancies between measurements and calculations.

\section{III.B. Phantom materials}

Phantoms should be made of a water-equivalent or known electron-density material so that the treatment planning system can accurately calculate dose to the phantom. A large number of such phantoms of different shapes made of waterequivalent materials are commercially available. When nonwater-equivalent materials such as PMMA and polystyrene are used, validation of the dose distribution calculation algorithm should be conducted before clinical use.

Additional considerations are required when radiographic film is used. Optical and UV light will expose the film, so the phantom must be light tight and internally opaque to prevent exposure by Čerenkov radiation. Radiographic film measurements are typically made in homogeneous phantoms. It is best to use a light-tight compression film phantom, although paper packaged (ready-pack) film use is acceptable without a light-tight phantom as long as the packaging is carefully vented or adjusted to prevent trapped air bubbles and the film package is compressed. The use of phantoms with $\mathrm{Pb}$ or other high atomic number materials is not recommended. ${ }^{158,159}$

\section{III.C. Phantom characterization}

The dimensions of a phantom should be verified prior to first use. IMRT treatment plan validations necessarily require the CT simulation of the phantom. These imaging data should also be used to inspect the construction of the phantom. Particular attention should be paid to flaws in the phantom material such as voids. Dosimeter positions should be identified on the CT scan study. This is especially important for anthropomorphic phantoms, where the dosimeter positions should be localized either by visualizing the dosimeter voids or with radiopaque dummy markers put in place of the dosimeters. For all phantoms, delineation of larger dosimeters, such as ionization chambers, is necessary to compute the volume-averaged dose. This can be done by imaging the phantom and contouring the detector volume in the treatment planning system as a structure of interest.

\section{DOSE DISTRIBUTION COMPARISONS AND EVALUATIONS}

This section describes published dose distribution comparison algorithms, their use, and their limitations when applied to IMRT. The measurements of complex dose distributions described in this report are of little value unless they can be quantitatively compared against calculated dose distributions. The value of quantitative dose distribution comparisons was first identified during the photon and electron treatment planning consortia of the 1990s. They developed a series of tools for comparing the 3D dose distributions produce by treatment planning systems and the 2D measured dose distributions (typically interpolated from water phantom data). ${ }^{160}$

With IMRT, the need for quantitative, efficient dose distribution comparison tools is even greater. IMRT requires accurate multidimensional validation by quantitative comparison between measurement and calculation.

\section{IV.A. Dose distribution registration}

Dose distribution comparisons are useful only if the two distributions have been accurately coregistered. Treatment planning systems calculate dose in 3D and will often export a planar dose distribution for comparison against measurement. To accurately compare calculated and measured dose distributions, the dose distribution positions should be presented in a common coordinate system.

The dose measurement phantom will have a method for alignment with respect to the linear accelerator. That system 
may be similar to systems used for patient alignment, namely, a set of radiopaque and visible markers that is identified on the treatment planning system prior to producing the test treatment plan or phantom plan. Localizing the dose measurement location for point-dose measurements (e.g., ionization chambers) may be as straightforward as using machined drawings of the phantom and knowledge of the center of the active chamber volume. For film dosimetry, the film should be accurately placed within the phantom and pin pricked or otherwise marked at known locations. This, however, is insufficient to characterize the measured dose distribution locations. A detailed understanding of the dose distribution comparison software is also necessary to accurately coregister the location of the dose measurement. Some dose distribution comparison software does not provide the capability of an independent registration of the calculated and measured dose distributions, but provides only relative registration. Relative registration involves selecting landmarks in the calculated and measured dose distributions, necessitating that fiducial landmarks be placed on the calculated dose distribution and either the film image or dose distribution. This is often not possible (for example, in phantoms where the phantom localization fiducial markers are placed on the outside of the phantom). In this case, care must be taken to assure that the localizations of the film and phantom coordinates are as independent as possible, as opposed to aligning the two dose distributions based on only the dose, which would be insensitive to an error in the dose distribution localization.

\section{IV.B. Dose comparison tools}

Van Dyk et al. ${ }^{161}$ were the first to publish on the importance of applying a particular dose distribution comparison test based on the local dose gradient. In regions of shallow dose gradient, the numerical differences between two compared dose distributions provide an effective tool in determining whether the two distributions agree. However, in steep dose gradient regions, the dose difference is very sensitive to small spatial offsets and can show misleadingly large discrepancies between the compared dose distributions. This sensitivity leads to the concept of distance-toagreement, where the distance between steep dose gradient regions is used as a determinate of dose distribution comparability [Fig. 4(e)]. The sensitivity of dose comparison tools as a function of local dose gradient has fostered development of other dose comparison tools as well, some of which are described in this report.

\section{IV.B.1. Dose distribution overlays}

A basic consideration of dose distribution comparisons is to make them quantitative, yet allow the observer an efficient evaluation of the results. Dose distribution overlays provide a rapid overview of the two dose distributions (Fig. 3 shows an example of superimposed isodose distributions). ${ }^{162}$ The individual dose distributions can be displayed as isodose contours (Fig. 3) or color bands. An isodose contour connects regions within a dose distribution that share a common

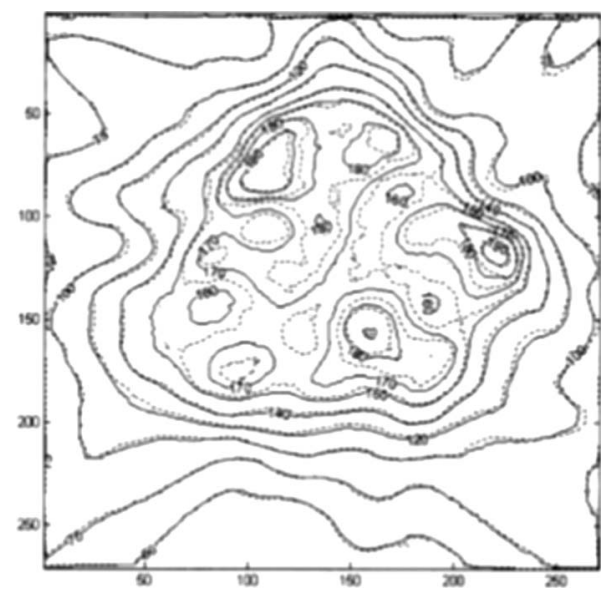

FIG. 3. Superimposed dose distributions intended to compare IMRT dose distributions. In this case, the solid and dashed lines are the calculated and measured distributions, respectively. When the superimposed isodoses overlap, the reviewer can readily see that they agree. However, when the doses differ, such as in the high-dose region in this example, a quantitative evaluation is difficult. Reference is from Childress et al. (Ref. 162).

dose level. Contour lines will cluster in steep dose gradient regions. Because isodose contours identify the location of a specific dose level, for a given dose uncertainty, variation in the contour position is greater in shallow than steep dose gradient regions, so isodose contours of superimposed dose distributions with constant dose distribution differences will lie farther apart in regions of shallow dose gradients than steep dose gradients. Typical graphical presentations of superimposed isodose distributions do not discriminate with respect to the local dose gradient, so such dose differences are challenging to interpret.

This sensitivity of the isodose line position with respect to the local dose gradient should be considered when superimposing two dose distributions. Often the selection of isodose lines can make quantitative evaluation more efficient. For example, selecting isodose lines that reflect the doses found in the steep dose gradient regions aids in the assessment of the relative locations of the gradients in the two dose distributions. Conversely, selection of isodose contours near the maximum dose, and consequently existing in shallow dose gradient regions, may yield isodose contours that do not appear to match due to the sensitivity of contour positioning with respect to small dose deviations. It is this feature that limits the utility of superimposed isodose distributions for dose distribution comparisons.

\section{IV.B.2. Dose-difference distributions}

The dose-difference tool displays the numerical difference between two dose distributions. Figure 4 shows examples of the dose-difference tool for the two dose distributions [Figs. 4(a) and 4(b), provided from Childress and Rosen ${ }^{162}$ ] shown in Fig. 3. In this case, both absolute [Fig. 4(c)] and relative [Fig. 4(d)] dose differences are shown. There are many striped regions that exceed 3\% in the periphery of the dose distributions. These types of striped dose discrepancies are often seen in cone-beam IMRT dose distributions. While the 


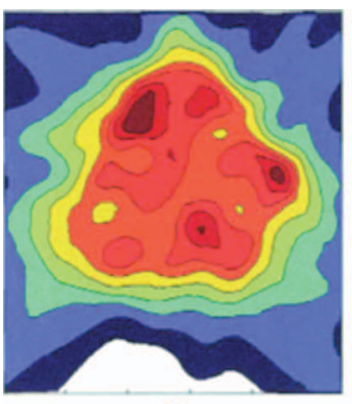

(a)

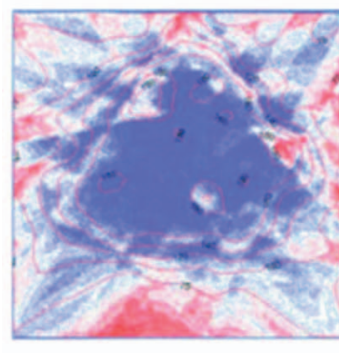

(c)

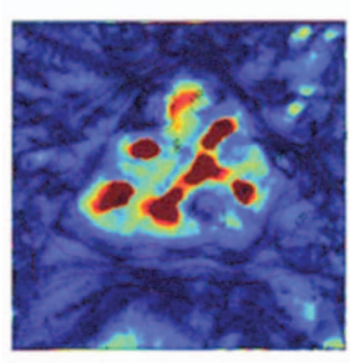

(e)

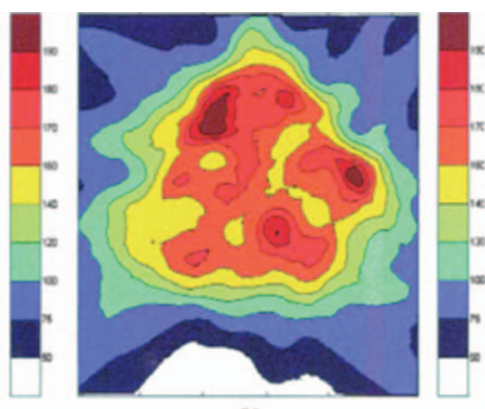

(b)
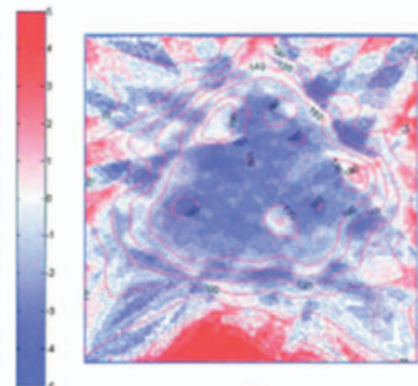

(d)

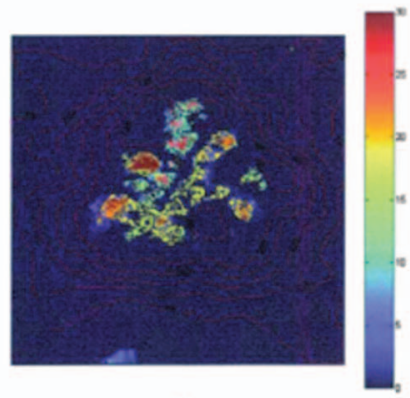

FIG. 4. Example of dose distribution evaluation and display tools. Examples are from Childress and Rosen (Ref. 162).

dose-difference tool yields a quantitative comparison between two dose distributions, it tends to be overly sensitive in steep dose gradient regions.

Figure 5 shows comparisons between two mathematically defined dose distributions ${ }^{163}$ that mimic $10 \times 10 \mathrm{~cm}^{2} 6 \mathrm{MV}$ fields. One field is left intact (the reference distribution), while the second (the evaluated distribution) is divided into four quadrants, each manipulated to highlight different characteristics of the dose evaluation tools. The evaluated distribution in quadrant 1 is identical to the reference distribution. The evaluated dose distribution in quadrant 2 has been scaled such that the dose varies by $\pm 6 \%$ at the field edges $\left(1.2 \% \mathrm{~cm}^{-1}\right.$ dose gradient). The evaluated dose distribution in quadrant 3 has been shifted by $1.2 \mathrm{~mm}$ for each $\mathrm{cm}$ of off-axis distance to mimic spatial discrepancies between dose distributions. A combination of the dose gradient and shift are applied in quadrant 4 . For quadrants $2-4$, the reference and evaluated dose distributions coincide along the center of the field edge. For these examples, the acceptable dose difference is $\pm 3 \%$.

The dose difference for the fields shown in Figs. 5(a) and 5(b) is shown in Fig. 5(c). In quadrant 1 (where both dose
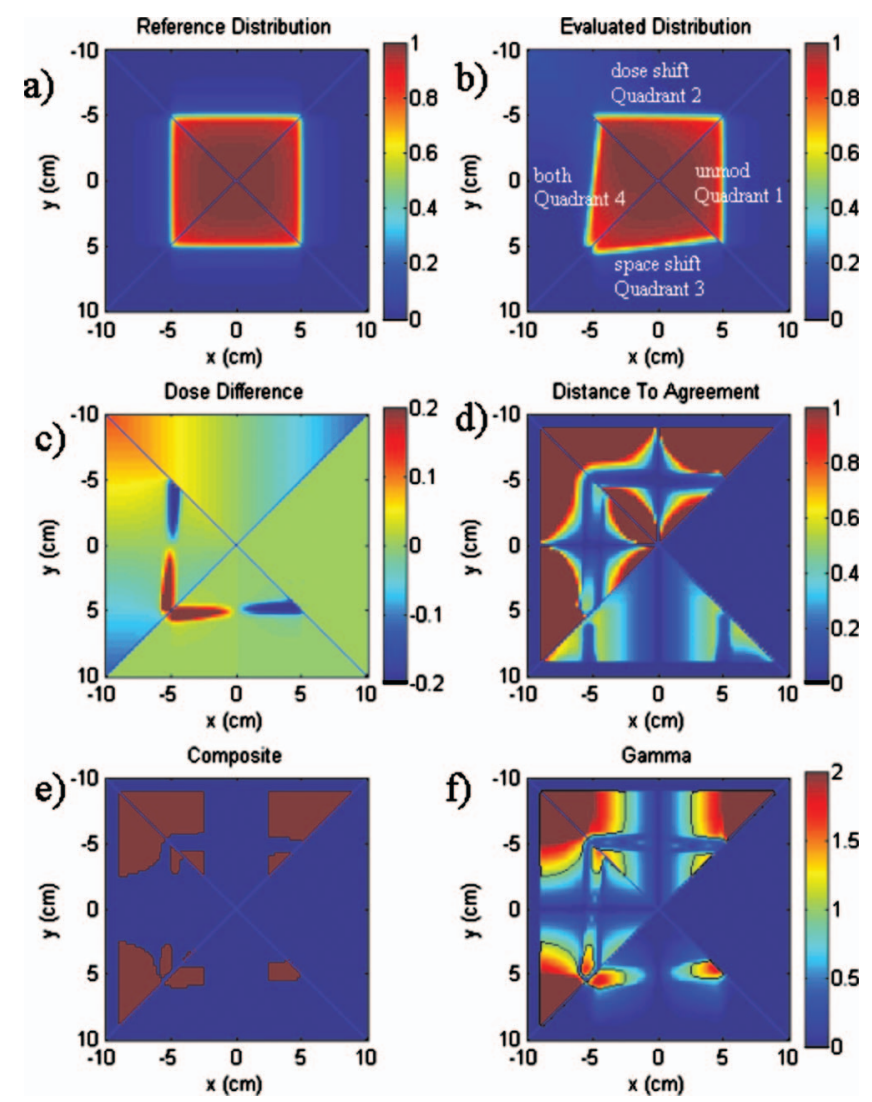

FIG. 5. Numerically defined dose distributions provided to illustrate dose distribution comparison tools. Examples are from Low and Dempsey (Ref. 163).

distributions are the same), the dose difference is uniformly zero. In quadrant 2 (dose gradient), the dose difference varies smoothly in both steep and shallow dose gradient regions. In quadrant 3 (shift), the dose difference becomes large $(> \pm 20 \%)$ even though the shifts are relatively small $(<6 \mathrm{~mm})$ because of the steep dose gradients. Even where the shift is $<2 \mathrm{~mm}$, the dose differences exceed $\pm 3 \%$. This highlights the sensitivity of the dose-difference tool in steep dose gradient regions.

\section{IV.B.3. Distance-to-agreement}

The DTA tool was developed to provide the user with a measurement of the distance discrepancy between two dose distributions. ${ }^{160,161}$ A typical application of the DTA tool is to calculate the DTA value for each reference point by scanning the evaluated distribution for the closest point that has the same dose value as the reference point. This is equivalent to determining the closest approach of the evaluated isodose curve with the same dose as the reference point. In steep dose gradient regions, this can be interpreted as the distance between the two dose distributions. This interpretation is based on the assumption that the distance is caused primarily by a spatial offset between the two distributions. For distributions that differ by such an offset, the DTA distribution provides an effective and accurate measurement of the offset. However, the discrepancy may not be due to an error of the 
dose calculation algorithm or delivery hardware, but simply due to experimental error. While as originally described, the DTA tool provides only the magnitude of the distance; the sign of the dose difference can be added to the DTA. This provides an indication of which of the two dose distributions is greater. It can also be used to quickly determine if the discrepancy is due to a spatial offset due to one distribution being "larger" than the other.

Figure 4(e) shows the DTA for the example provided by Childress and Rosen. ${ }^{162}$ In this case, the regions of large DTA are located in the high-dose region, where the dose gradient is shallow. Figure 5(d) shows the DTA for the example from Low and Dempsey. ${ }^{163}$ For this example, the DTA criterion is $3 \mathrm{~mm}$. The DTA in quadrant 1 is zero, while in quadrant 2, the DTA is small and large in steep and shallow dose gradient regions, respectively. The DTA becomes greater than $3 \mathrm{~mm}$ in the region where the dose discrepancy is $\leq 1 \%$, except in the steep dose gradient region. The DTA tool is very sensitive in the shallow dose gradient regions, but insensitive to even large dose differences $(>6 \%)$ in steep dose gradient regions where the spatial discrepancy of the dose distributions is small. In quadrant 3 , the DTA increases as expected with the applied spatial dose shift of the evaluated distribution. Because shallow dose gradient regions dominate clinical dose distributions, the DTA tool typically yields large regions where the DTA value is large but these large values are not clinically relevant. This makes visual interpretation of DTA distributions challenging.

\section{IV.B.4. Quantitative comparison tools}

The dose overlay, dose difference, and DTA distributions provide good comparisons between the two distributions. The remaining tools to be discussed require that the user specifies acceptance or scaling factors that are used in the comparisons.

IV.B.4.a. Composite tool. Due to the complementary sensitivity of dose-difference and DTA tools, the composite tool was developed ${ }^{160}$ to identify regions that disagreed by both dose and distance. Acceptance criteria for the dose difference and DTA are selected and the regions that exceed the criteria in both dose difference and DTA are identified. These points are said to fail the comparison, while the remaining points pass and can be displayed as a binary distribution. Figure 5(e) shows an example of the composite tool for the case shown by Low and Dempsey ${ }^{163}$ using dose and distance criteria of $3 \%$ and $3 \mathrm{~mm}$, respectively. In quadrant 1 , the composite tool passes in all locations. In quadrant 2 , the composite tool passes when the dose difference is less than $3 \%$ or in the steep dose gradient regions, where the DTA criterion passes. In quadrant 3 , the composite distribution passes except for the steep dose gradient region where the applied shift is $>3 \mathrm{~mm}$. Quadrant 4 shows failures that encompass the regions shown in quadrants 2 and 3.

IV.B.4.b. Gamma and similar tools. While the composite tool indicates the location of failure, it provides only a pass/ fail indication. There is no indication of the magnitude of

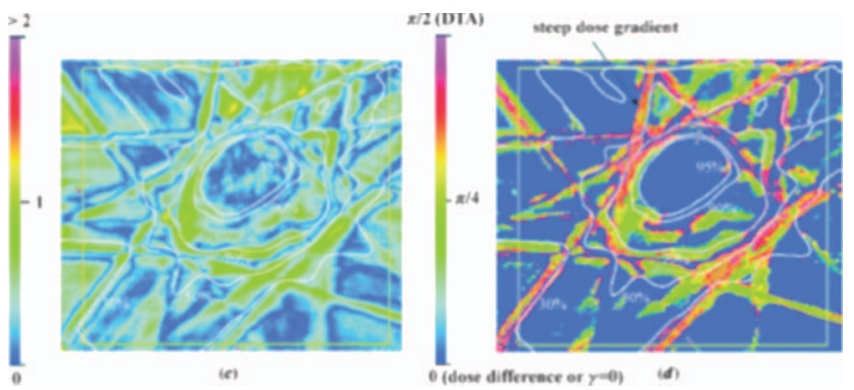

FIG. 6. Example of the use of the $\gamma$ and $\gamma$ angle tools is from Stock et al. (Ref. 164).

failure. Having a quantitative measure of the degree of agreement allows the user to develop a response appropriate to the degree of disagreement.

Low et al. ${ }^{163,241}$ developed a tool that combines dose and distance criteria in a single, quantitative test. The doses and spatial coordinates are first renormalized by user-selected dose and distance acceptance criteria. Because the resulting quantities are unitless, they can be evaluated simultaneously. The two dose distributions to be compared are assigned as the reference and evaluated distribution. For each point in the reference distribution, the normalized distance to each point in the evaluated distribution is measured, where the distance includes both normalized spatial and dose values. The closest approach (shortest distance) of the reference distribution is identified as the $\gamma$ value. The $\gamma$ value is unity when the closest approach of the reference distribution is on the unit sphere. The unit sphere indicates the region within which the comparison test passes. Therefore, if the reference distribution pierces the unit sphere, the $\gamma$ test passes, otherwise it fails. The $\gamma$ value can be displayed as a distribution for evaluation. Figure 5(f) shows an example of the $\gamma$ distribution for the two dose distributions shown in Figs. 5(a) and 5 (b), with scaling criteria of $3 \mathrm{~mm}$ and $3 \%$. As a guide to the eye, a contour is provided at the value of $\gamma=1$. The value of $\gamma$ is zero in quadrant 1 . In quadrant 2 , the dose difference exceeds $3 \%$ at an off-axis distance of $2.5 \mathrm{~cm}$ and this is evident by the $\gamma=1$ contour. The value of $\gamma<1$ in the steep dose gradient region is because the DTA is less than $3 \mathrm{~mm}$ in this region. The value of $\gamma$ is dominated in this region by the distance between the reference and the evaluated distributions. In quadrant $3, \gamma$ is dominated throughout most of the dose distribution by relatively small dose differences. This is true except within the steep dose gradient region, where the dose differences are large and the value of $\gamma$ is determined by the spatial distance between the reference and the evaluated distributions. The $\gamma=1$ contour appears in the region where the spatial shift exceeds $3 \mathrm{~mm}$ (the distance criterion).

A clinical example of the use of the tool is presented in Fig. 6 (Ref. 164) for the head-and-neck case. Figure 6(a) shows the value of $\gamma$ with the superimposed calculated distribution. The example shows the regions that fail the criteria (3\% and $3 \mathrm{~mm}$ in this case), highlighting areas of significant failure. Stock et al. ${ }^{164}$ also identified the fact that the angle that the $\gamma$ vector makes to the spatial axes (the complement 

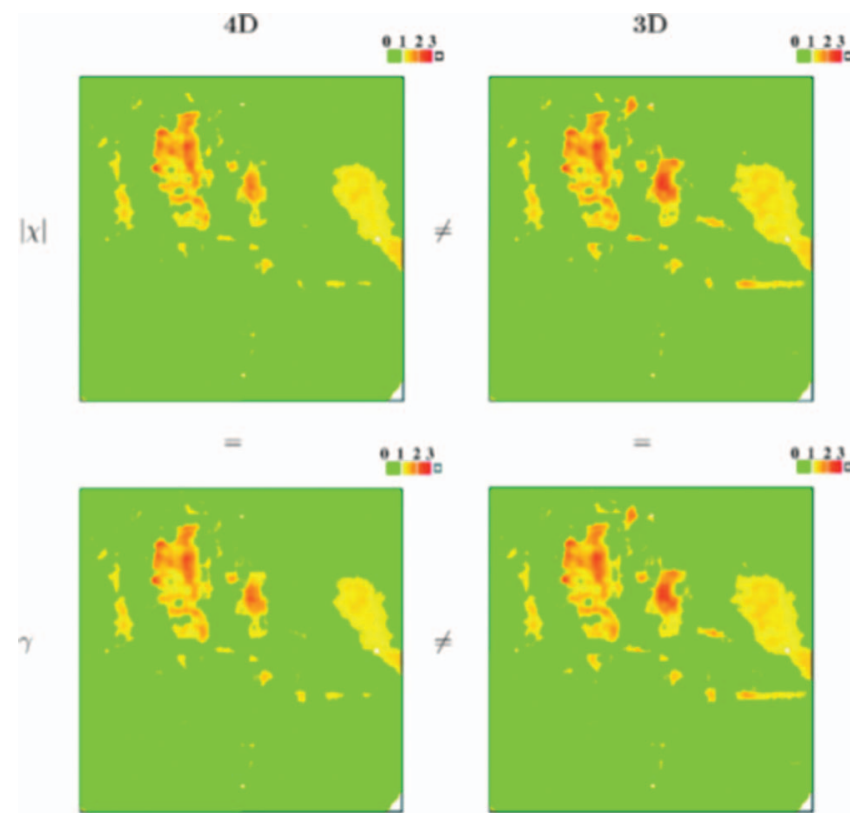

FIG. 7. Comparison between $\gamma$ and $\chi$ as defined by Bakai et al. (Ref. 175).

to the angle the vector makes to the dose axis) can be used to determine whether the $\gamma$ discrepancy is due to a spatial or dose error. An example of the $\gamma$ angle is shown in Fig. 6(b). ${ }^{164}$ While the $\gamma$ angle may be useful in evaluating the failure reasons, it is very sensitive to dose distribution noise and can be difficult to visually evaluate.

The $\gamma$ tool has been successfully implemented in a number of dose comparison studies, ${ }^{12,123,133,165-174}$ and some authors have proposed modifications to the tool to provide more efficient calculations and to extend the capabilities of the tool. ${ }^{162,164,167,175-178}$ One example is shown in Fig. 4(f), provided from Childress and Rosen ${ }^{162}$ based on their normalized agreement test (NAT). The NAT calculation is similar to gamma, but is zero where the doses agree within acceptable tolerances, so the comparison values are displayed only in the regions that fail the comparison and are greater than $75 \%$ of the prescription dose. This allows the user to focus on the regions that failed and not be distracted by the regions that passed the comparison tests.

One of the difficulties with the use of the $\gamma$ tool had been the relatively large computation time and the requirement for interpolation of the evaluated distribution when measurements are widely spaced relative to the DTA criterion. Bakai et al. ${ }^{175}$ proposed to use the acceptable local dose error and a function called $\chi$, which is computed more efficiently than $\gamma$, but yields very similar information. Figure 7 shows an example from Bakai et al. ${ }^{175}$ where they compared a measured and calculated dose distribution. They conducted both the $\gamma$ and $\chi$ tests in two and three spatial dimensions (the measured distribution was film, but the calculated distribution was in three dimensions). The two-dimensional evaluation was unable to evaluate nearby out-of-plane points and therefore overestimated both $\gamma$ and $\chi$. They concluded that, when possible, the calculated dose distribution should be queried in all three dimensions. Because $\chi$ was signed, it was capable of assisting in the evaluation of two shifted dose distributions, although the definition of $\gamma$ can be slightly modified to account for the sign of the dose difference. A new, recursive method for computing $\gamma$ was recently developed by Ju et al., that significantly improves $\gamma$ computation, speed, and accuracy. $^{240}$

Another tool uses a gradient-compensation method. ${ }^{179}$ The local dose gradient for each point in a distribution is calculated in $2 \mathrm{D}$ or $3 \mathrm{D}$. Then, the user selects a distance parameter (typically $1 \mathrm{~mm}$ ) to account for potential errors due to geometric uncertainties such as film alignment and calculation grid size (e.g., one-half of the grid size). The dose gradient at each point is then multiplied by the distance parameter to determine the gradient compensated distribution. Dose differences that are not explained by the gradient compensation remain and are displayed with an isodose color wash. This method is used in conjunction with standard dose evaluation methods to highlight differences that may be due to systematic discrepancies between calculations and measurements.

\section{IV.C. Summary}

No single dose comparison tool provides all of the information necessary to quantitatively evaluate or compare dose distributions. Each tool has limitations that need to be understood when conducting evaluations. The most basic dose overlay method requires that the user interpret the differences themselves, relying on visually detecting regions where the doses differ. The dose-difference tool is a very intuitive tool, but has the limitation that very large dose differences can be caused by relatively small spatial discrepancies in steep dose gradient regions including coregistration errors. Given its quantitative and intuitive nature, however, the dose-difference tool should be employed when other tools, such as the $\gamma$ tool, indicate a discrepancy. Tools, such as the $\gamma$ tool, that integrate more than one type of discrepancy evaluation are useful when a large amount of dose data needs to be reviewed quickly, such as for routine patient QA. When discrepancies are identified, the clinical impact of those discrepancies can be determined using the dosedifference tool. Dose analysis tools such as $\gamma$ and the gradient tool should be used to evaluate measurements compared to $3 \mathrm{D}$ calculations for completeness. These analysis tools are best applied to data with limited noise.

\section{ADDITIONAL SYSTEMS FOR QUALITY ASSURANCE}

\section{V.A. Electronic portal imaging devices}

EPIDs have been designed for verification of patient position and are mounted to the treatment gantry. However, the ability to acquire 2D electronic data in the machine geometry have led to the investigation of EPIDs for verification of individual leaf position, ${ }^{180,181}$ radiation vs light field checks, and IMRT field verification. Commercial EPIDs include charge-coupled camera devices (CCD), scanning liquid ionization chamber, and active matrix flat panel imagers (AM- 
FPIs). Recent investigations have focused primarily on applications with AMFPIs. van Elmpt et al. ${ }^{182}$ reviewed the different approaches for using EPIDs for radiation therapy dosimetry applications. The primary investigations have been for EPID systems incorporating a scintillator where indirect detectors are used. ${ }^{183-186}$ Corrections are made to a system that has been optimized for imaging. Additional investigations have been conducted for prototype and systems modified from a commercial configuration where there is no scintillator above the detector and measurements are made in a direct detection mode. ${ }^{187-190}$

\section{V.A.1. Application to IMRT}

EPIDs offer the potential to save time when compared to film dosimetry. They offer a finer spatial resolution than array devices. However, the application of EPIDs to IMRT is limited to measurements of individual fields and EPIDs cannot be placed in a solid water stack. For all of the EPID systems, a number of corrections must be applied to convert the resulting signal into fluence or dose in the detector.

EPIDs have been used for pretreatment and transit dosimetry applications. In the pretreatment configuration, the expected response of the EPID is typically determined at the plane of the detector as a portal dose image. ${ }^{183-186}$ The response of the EPID is then converted to dose and compared to the predicted dose image. Quantitative dosimetric measurements for IMRT fields have also been made with systems using a direct detection mode. ${ }^{187,190}$ For clinical dosimetry applications, the system needs to be validated against reference dosimeters of appropriate accuracy and resolution. The other application of EPIDs is for transit dosimetry where measurements are made during a patient's treatment and used to evaluate the accuracy of the delivery considering the patient's geometry. ${ }^{191-193}$ The use of EPIDs for transit dosimetry continues to be an important research topic with the potential for providing important real-time information about the accuracy of the delivery of IMRT treatments.

\section{V.A.2. Recommendations for use}

Once an IMRT program has been started with ion chamber and film measurements, it may be appropriate to use an EPID for individual IMRT field verification measurements if a reliable method of operation has been developed. The EPID response must be characterized for a range of situations (e.g., dose, dose rate, field size, and leaf speed). Once the system is characterized, a number of corrections must be made to the system depending on the type of system and the composition of the detector. The presence of a fluorescent screen leads to an over-response of the detectors to low doses. To calculate a portal dose prediction or portal dose image for AMFPI systems, pencil beam, ${ }^{185}$ convolution, ${ }^{183}$ or Monte Carlo ${ }^{194}$ techniques have been used to approximate or model the interactions in the EPID including the effect of the fluorescent layer.

Some centers are utilizing commercial systems for IMRT dosimetry. When establishing a QA program with an EPID, the sensitivity of the system and the appropriate action levels and criteria for evaluation must be set. ${ }^{195}$ Further development of EPIDs for individual IMRT field verification and for patient transit dosimetry is expected to continue. ${ }^{191-193,196-200}$ Exciting developments include reconstruction of threedimensional dose distributions. ${ }^{201}$

\section{V.B. Three-dimensional detectors}

Truly comprehensive dosimetric verification of IMRT requires a dosimetry system with full capability in 3D. Traditional 2D verification techniques (e.g., film), and the more recent 2D array detectors discussed above, present at best a partial sampling of the whole distribution. Partial sampling may be adequate as a routine check for an IMRT program that has extensive prior validation. In the general case, however, and in particular for the commissioning of a new IMRT technique, an extensive 3D dosimetry technique is highly desirable. The criticality of this issue is exemplified by a recent report from the Radiological Physics Center (RPC), ${ }^{202}$ which revealed that between 2001-2009, more than 350 institutions failed to pass the head-and-neck IMRT credentialing phantom test on the first attempt, despite generous criteria (7\% dose difference and $4 \mathrm{~mm}$ distance-to-agreement). The RPC criteria were applied to TLD measurements at six points inside the simulated planning-target-volume (PTV) and GAFCHROMIC ${ }^{\circledR}$ film measurement in a single axial plane through PTV and organ-at-risk. The question ariseswhat percentage of institutions would have failed if a more comprehensive 3D measurement had been feasible rather than measurements restricted to the central film plane and TLD points? This question can only be adequately answered if the measurement is a comprehensive verification in 3D and presents a compelling argument for the need for a clinical 3D dosimetry system. A relatively new technology with the capacity to address this need and provide high-resolution, accurate 3D dosimetry has emerged under the initial name of "gel-dosimetry." In this report, we will use the term "3D dosimetry" to reflect the fact that some new 3D dosimeters are no longer gel-based.

A goal of 3D dosimetry has been formulated in the resolution-time-accuracy-precision (RTAP) criteria. $^{203}$ The RTAP ideal functionality incorporates a spatial resolution of $1 \mathrm{~mm}^{3}$, short read-out time of $\ll 1 \mathrm{~h}$, accuracy within $3 \%$ of true value, and a noise level within $1 \%$. Several dosimetry systems are approaching this goal, and the field of 3D dosimetry continues to accelerate in innovation and promise. Recently, new 3D dosimetry materials have been proposed with striking performance characteristics. ${ }^{204,205}$ The three principal categories of 3D dosimeters are polyacrylamide (PAG) gels, ${ }^{206}$ Fricke gels, ${ }^{204}$ and radiochromic plastics, ${ }^{205}$ and the two main methods of dose-readout are magnetic resonance (MR) imaging ${ }^{207,208}$ and optical-computedtomography (optical-CT). ${ }^{203,204,209}$ Other readout techniques are under evaluation, including $\mathrm{x}$-ray-CT and ultrasound, but are not discussed further here as they are more preliminary.

\section{V.B.1. Polymerizing gels}

The original PAG gel ${ }^{206}$ consisted of bis (3\%), acrylamide $(3 \%)$, nitrogen, and gelatin $(5 \%)$ by weight. Newer formula- 
tions are more radiation sensitive $\mathrm{e}^{210}$ and can be made under conditions of normal oxygenation. ${ }^{210}$ PAGs utilize the mechanism of radiation-induced polymerization of monomers, where small monomer molecules join together under the influence of ionizing radiation. ${ }^{211}$ The resultant polymer microparticles are fixed in the gelatin lattice, yielding a stable impression of the dose. Below saturation, regions of the gel exposed to ionizing radiation exhibit polymerization in proportion to the dose, and the polymerization affects both the MR relaxivity of water protons and also the optical scattering power of the gel. High resolution 3D maps of dose have been achieved both by MR scanning and optical-CT scanning (Sec. V B 3). Initial applications to IMRT verification have been promising. ${ }^{64,210,212-217}$

\section{V.B.2. Fricke and radiochromic gels and plastics}

The first gel dosimeter arose from the work of Gore et al., ${ }^{218}$ who proposed that the well established liquid Fricke dosimeter ${ }^{219,220}$ could be gelated and probed by nuclear magnetic resonance imaging (MRI) rather than the conventional spectrophotometry. The addition of a stabilizing gelatin matrix to the aqueous Fricke solution created the first gel dosimeter, where 3D MRI images of the $T 1$ relaxation parameter showed the relaxation rate $1 / T 1$ proportional to the absorbed dose. A limitation of gelatin Fricke dosimeters is that the radiation-induced ferric ions diffuse through the gel matrix, leading to degradation and eventual loss of the recorded dose distribution with time..$^{221,222}$ The practical implication is that Fricke gel dosimeters must be scanned within $2 \mathrm{~h}$ postirradiation, presenting logistical difficulties in a clinical setting. Most Fricke gel dosimetry has utilized MR as the readout method; however, Kelly et al. $^{204}$ introduced xylenol-orange dye to create a Fricke gel compatible with optical-CT imaging. Recently, a promising new radiochromic material, PRESAGE ${ }^{223}$ has been introduced and detailed studies of the basic dosimetric properties have confirmed its promise for 3D dosimetry. ${ }^{224}$ Principal advantages include relative insensitivity of dose response to atmospheric exposure and to the nature of radiochromic optical contrast that is light absorbing rather than light-scattering (peak OD change is at $\sim 633 \mathrm{~nm}$ ). The absorptive nature of the contrast is more amenable to accurate dose read-out by opticalcomputed-tomography optical-CT. ${ }^{225-227}$

\section{V.B.3. Readout techniques}

To date, most gel dosimetry has utilized MR scanning, which can yield excellent results, provided sufficient care is taken to characterize the MR scanner and implement appropriate sequence protocols..$^{64,207,228-230}$ In particular, attention is required to minimize uncertainties that originate from the technical challenges of controlling magnetic field uniformity and gradients with sufficient precision, avoiding MRI sequences that cause excessive temperature increase associated with RF energy deposited in the gel during MR imaging. ${ }^{228}$ For MR measurements requiring high accuracy and highspatial resolution, long imaging times (several hours) may be required to achieve the low uncertainty and high spatial res- olution associated with standard dosimeters. An alternative readout technique, optical-CT, ${ }^{166,203,204,209,227,231-235}$ has been proposed for imaging PAG gels; an approach analogous to first-generation $\mathrm{x}$-ray CT. In optical-CT the $\mathrm{X}$-ray source is replaced by a visible laser and the $\mathrm{x}$-ray detector replaced with a light-sensitive photodiode. Resonstructed images of optical attenuation coefficients are proportional to dose and can be converted to dose maps using a calibration curve. The high proportion of scatter in PAGs restricts optical-CT to slow first-generation scanning-laser configurations. Much faster broad-beam and cone-beam configurations have been developed for radiochronic gels and plastics, where the scatter component is much less. ${ }^{232,237}$ For these approaches corrections may be necessary for spectral warping ${ }^{238}$ and residual scatter. ${ }^{232,239}$

\section{V.B.4. Recommendations for use}

The 3D dosimetry techniques described here are still in the developmental stage and should be embarked upon with that understanding in mind. Commercial support for these techniques, while expanding, is still limited. At present, the only commercially available system is the BANG gel system from MGS Research. This polymer-gel/optical-CT readout system has been shown to be effective $\mathrm{e}^{166,217,236}$ and is available as a remote dosimetry service by the gel manufacturer. Detailed specifications on the accuracy of system performance of the remote system are not available at present. There are other dosimetry materials and readout techniques that can be employed. Each has strengths, but also areas where care must be taken to get adequate results. If MR scanning is to be used, the performance and sequences of the MR scanning system should be investigated first. Similarly, the performance characteristics of optical-CT systems need to be thoroughly evaluated prior to use. For all present 3D materials, it is important that the dose calibration curve is generated from the same batch and undergoes the same thermal and temporal histories as the experimental dosimeter.

\section{SUMMARY}

This report provides information to the physicist regarding the proper applications of different dosimeters, phantoms, and analysis techniques for IMRT dose distributions. The detectors and phantoms used in commissioning an IMRT program are frequently not the same systems used for pretreatment quality assurance applications. This report also provides guidance on the potential pitfalls by highlighting areas where certain systems are inappropriate for use. When establishing a new IMRT program, this information should be used in conjunction with other guidance documents on IMRT.

\footnotetext{
${ }^{a)}$ Present address: UCLA, Los Angeles, CA 90024. Electronic mail: dlow@mednet.ucla.edu

${ }^{1}$ B. Fraass, K. Doppke, M. Hunt, G. Kutcher, G. Starkschall, R. Stern, and J. Van Dyke, "American Association of Physicists in Medicine Radiation Therapy Committee Task Group 53: Quality assurance for clinical radiotherapy treatment planning," Med. Phys. 25, 1773-1829 (1998).

${ }^{2}$ F. M. Khan, The Physics of Radiation Therapy, 3rd ed. (Lippincott Williams \& Wilkins, Philadelphia, 2003).
} 
${ }^{3}$ G. J. Kutcher et al., "Comprehensive QA for radiation oncology: Report of AAPM Radiation Therapy Committee Task Group 40," Med. Phys. 21, 581-618 (1994).

${ }^{4}$ J. Zhu, F. F. Yin, and J. H. Kim, "Point dose verification for intensity modulated radiosurgery using Clarkson's method," Med. Phys. 30, 22182221 (2003).

${ }^{5}$ A. Boyer, L. Xing, C. M. Ma, B. Curran, R. Hill, A. Kania, and A. Bleier, "Theoretical considerations of monitor unit calculations for intensity modulated beam treatment planning," Med. Phys. 26, 187-195 (1999).

${ }^{6} \mathrm{Z}$. Chen, L. Xing, and R. Nath, "Independent monitor unit calculation for intensity modulated radiotherapy using the MIMiC multileaf collimator," Med. Phys. 29, 2041-2051 (2002).

${ }^{7}$ J. H. Kung, G. T. Chen, and F. K. Kuchnir, "A monitor unit verification calculation in intensity modulated radiotherapy as a dosimetry quality assurance," Med. Phys. 27, 2226-2230 (2000).

${ }^{8}$ J. S. Tsai, M. J. Engler, and J. Liu, "Quasi-independent monitor unit calculation for intensity modulated sequential tomotherapy," J. Appl. Clin. Med. Phys. 3, 135-153 (2002).

${ }^{9}$ L. Xing, Y. Chen, G. Luxton, J. G. Li, and A. L. Boyer, "Monitor unit calculation for an intensity modulated photon field by a simple scattersummation algorithm," Phys. Med. Biol. 45, N1-N7 (2000).

${ }^{10}$ X. Chen, N. J. Yue, W. Chen, C. B. Saw, D. E. Heron, D. Stefanik, R. Antemann, and M. S. Huq, "A dose verification method using a monitor unit matrix for dynamic IMRT on Varian linear accelerators," Phys. Med. Biol. 50, 5641-5652 (2005).

${ }^{11}$ J. M. Galvin, G. Ezzell, A. Eisbrauch, C. Yu, B. Butler, Y. Xiao, I. Rosen, J. Rosenman, M. Sharpe, L. Xing, P. Xia, T. Lomax, D. A. Low, and J. Palta, "Implementing IMRT in clinical practice: A joint document of the American Society for Therapeutic Radiology and Oncology and the American Association of Physicists in Medicine," Int. J. Radiat. Oncol., Biol., Phys. 58, 1616-1634 (2004).

${ }^{12}$ Y. Yang, L. Xing, J. G. Li, J. Palta, Y. Chen, G. Luxton, and A. Boyer, "Independent dosimetric calculation with inclusion of head scatter and MLC transmission for IMRT,” Med. Phys. 30, 2937-2947 (2003).

${ }^{13}$ G. A. Ezzell, J. M. Galvin, D. Low, J. R. Palta, I. Rosen, M. B. Sharpe, P. Xia, Y. Xiao, L. Xing, and C. X. Yu, "Guidance document on delivery, treatment planning, and clinical implementation of IMRT: Report of the IMRT Subcommittee of the AAPM Radiation Therapy Committee," Med. Phys. 30, 2089-2115 (2003).

${ }^{14}$ L. Dong, J. Antolak, M. Salehpour, K. Forster, L. O’Neill, R. Kendall, and I. Rosen, "Patient-specific point dose measurement for IMRT monitor unit verification,” Int. J. Radiat. Oncol., Biol., Phys. 56, 867-877 (2003).

${ }^{15}$ D. A. Low, "Quality assurance of intensity-modulated radiotherapy," Semin. Radiat. Oncol. 12, 219-228 (2002).

${ }^{16}$ I. J. Das, C. W. Cheng, R. J. Watts, A. Ahnesjo, J. Gibbons, X. A. Li, J. Lowenstein, R. K. Mitra, W. E. Simon, and T. C. Zhu, "Accelerator beam data commissioning equipment and procedures: Report of the TG-106 of the Therapy Physics Committee of the AAPM," Med. Phys. 35, 41864215 (2008).

${ }^{17}$ G. A. Ezzell, J. W. Burmeister, N. Dogan, T. J. LoSasso, J. G. Mechalakos, D. Mihailidis, A. Molineu, J. R. Palta, C. R. Ramsey, B. J. Salter, J. Shi, P. Xia, N. J. Yue, and Y. Xiao, "IMRT commissioning: Multiple institution planning and dosimetry comparisons, a report from AAPM Task Group 119," Med. Phys. 36, 5359-5373 (2009).

${ }^{18}$ E. E. Klein, J. Hanley, J. Bayouth, F. F. Yin, W. Simon, S. Dresser, C. Serago, F. Aguirre, L. Ma, B. Arjomandy, C. Liu, C. Sandin, and T. Holmes, "Task Group 142 report: Quality assurance of medical accelerators," Med. Phys. 36, 4197-4212 (2009).

${ }^{19}$ D. A. Low, S. Mutic, J. F. Dempsey, R. L. Gerber, W. R. Bosch, C. A. Perez, and J. A. Purdy, "Quantitative dosimetric verification of an IMRT planning and delivery system," Radiother. Oncol. 49, 305-316 (1998).

${ }^{20}$ C. Martens, C. De Wagter, and W. De Neve, "The value of the PinPoint ion chamber for characterization of small field segments used in intensitymodulated radiotherapy," Phys. Med. Biol. 45, 2519-2530 (2000).

${ }^{21}$ M. Bucciolini, F. B. Buonamici, S. Mazzocchi, C. De Angelis, S. Onori, and G. A. Cirrone, "Diamond detector versus silicon diode and ion chamber in photon beams of different energy and field size," Med. Phys. 30, 2149-2154 (2003).

${ }^{22}$ W. U. Laub and T. Wong, "The volume effect of detectors in the dosimetry of small fields used in IMRT," Med. Phys. 30, 341-347 (2003).

${ }^{23}$ L. B. Leybovich, A. Sethi, and N. Dogan, "Comparison of ionization chambers of various volumes for IMRT absolute dose verification," Med. Phys. 30, 119-123 (2003).
${ }^{24}$ D. A. Low, P. Parikh, J. F. Dempsey, S. Wahab, and S. Huq, "Ionization chamber volume averaging effects in dynamic intensity modulated radiation therapy beams," Med. Phys. 30, 1706-1711 (2003).

${ }^{25} \mathrm{H}$. Bouchard and J. Seuntjens, "Ionization chamber-based reference dosimetry of intensity modulated radiation beams," Med. Phys. 31, 24542465 (2004).

${ }^{26}$ R. Capote, F. Sanchez-Doblado, A. Leal, J. I. Lagares, R. Arrans, and G. H. Hartmann, "An EGSnrc Monte Carlo study of the microionization chamber for reference dosimetry of narrow irregular IMRT beamlets," Med. Phys. 31, 2416-2422 (2004).

${ }^{27}$ K. A. Paskalev, J. P. Seuntjens, H. J. Patrocinio, and E. B. Podgorsak, "Physical aspects of dynamic stereotactic radiosurgery with very small photon beams (1.5 and $3 \mathrm{~mm}$ in diameter)," Med. Phys. 30, 111-118 (2003).

${ }^{28}$ F. Sánchez-Doblado, P. Andreo, R. Capote, A. Leal, M. Perucha, R. Arráns, L. Núñez, E. Mainegra, J. I. Lagares, and E. Carrasco, "Ionization chamber dosimetry of small photon fields: A Monte Carlo study on stopping-power ratios for radiosurgery and IMRT beams," Phys. Med. Biol. 48, 2081-2099 (2003).

${ }^{29}$ F. Sánchez-Doblado, R. Capote, A. Leal, J. V. Roselló, J. I. Lagares, R. Arráns, and G. H. Hartmann, "Microionization chamber for reference dosimetry in IMRT verification: Clinical implications on OAR dosimetric errors," Phys. Med. Biol. 50, 959-970 (2005).

${ }^{30}$ F. Sánchez-Doblado, R. Capote, J. V. Roselló, A. Leal, J. I. Lagares, R. Arráns, and G. H. Hartmann, "Micro ionization chamber dosimetry in IMRT verification: Clinical implications of dosimetric errors in the PTV," Radiother. Oncol. 75, 342-348 (2005).

${ }^{31}$ L. J. Humphries and J. A. Purdy, in Advances in Radiation Oncology Physics Dosimetry, Treatment Planning, and Brachytherapy, AAPM Monograph Vol. 19, edited by J. A. Purdy (AAPM, 1992).

${ }^{32} \mathrm{M}$. Westermark, J. Arndt, B. Nilsson, and A. Brahme, "Comparative dosimetry in narrow high-energy photon beams," Phys. Med. Biol. 45, 685702 (2000).

${ }^{33}$ P. D. Higgins, P. Alaei, B. J. Gerbi, and K. E. Dusenbery, "In vivo diode dosimetry for routine quality assurance in IMRT," Med. Phys. 30, 31183123 (2003).

${ }^{34}$ I. Griessbach, M. Lapp, J. Bohsung, G. Gademann, and D. Harder, "Dosimetric characteristics of a new unshielded silicon diode and its application in clinical photon and electron beams," Med. Phys. 32, 3750-3754 (2005).

${ }^{35} \mathrm{C}$. McKerracher and D. I. Thwaites, "Assessment of new small-field detectors against standard-field detectors for practical stereotactic beam data acquisition," Phys. Med. Biol. 44, 2143-2160 (1999).

${ }^{36} \mathrm{C}$. Li, L. S. Lamel, and D. Tom, "A patient dose verification program using diode detectors," Med. Dosim. 20, 209-214 (1995).

${ }^{37}$ T. Wolff, S. Carter, K. A. Langmack, N. I. Twyman, and P. P. Dendy, "Characterization and use of a commercial n-type diode system," Br. J. Radiol. 71, 1168-1177 (1998).

${ }^{38} \mathrm{X}$. R. Zhu, "Entrance dose measurements for in-vivo diode dosimetry: Comparison of correction factors for two types of commercial silicon diode detectors," J. Appl. Clin. Med. Phys. 1, 100-107 (2000).

${ }^{39} \mathrm{G}$. Rikner and E. Grusell, "General specifications for silicon semiconductors for use in radiation dosimetry," Phys. Med. Biol. 32, 1109-1117 (1987).

${ }^{40}$ B. Nilsson, B. I. Ruden, and B. Sorcini, "Characteristics of silicon diodes as patient dosimeters in external radiation therapy," Radiother. Oncol. 11, 279-288 (1988).

${ }^{41}$ S. N. Rustgi, "Evaluation of the dosimetric characteristics of a diamond detector for photon beam measurements," Med. Phys. 22, 567-570 (1995).

${ }^{42}$ S. N. Rustgi and D. M. Frye, "Dosimetric characterization of radiosurgical beams with a diamond detector," Med. Phys. 22, 2117-2121 (1995).

${ }^{43}$ C. De Angelis, S. Onori, M. Pacilio, G. A. Cirrone, G. Cuttone, L. Raffaele, M. Bucciolini, and S. Mazzocchi, "An investigation of the operating characteristics of two PTW diamond detectors in photon and electron beams," Med. Phys. 29, 248-254 (2002).

${ }^{44}$ M. Benabdesselam, B. Serrano, P. Iacconi, F. Wrobel, D. Lapraz, J. Herault, and J. E. Butler, "Thermoluminescence properties of CVD diamond for clinical dosimetry use," Radiat. Prot. Dosim. 120, 87-90 (2006).

${ }^{45}$ C. De Angelis, M. Bucciolini, M. Casati, I. Lovik, M. Bruzzi, S. Lagomarsino, S. Sciortino, and S. Onori, "Improvements in CVD diamond properties for radiotherapy dosimetry," Radiat. Prot. Dosim. 120, 38-42 (2006). 
${ }^{46}$ B. Marczewska, P. Olko, M. Nesladek, M. P. Waligorski, and Y. Kerremans, "CVD diamonds as thermoluminescent detectors for medical applications," Radiat. Prot. Dosim. 101, 485-488 (2002).

${ }^{47}$ M. Bruzzi, M. Bucciolini, M. Casati, C. DeAngelis, S. Lagomarsino, I. Lovik, S. Onori, and S. Sciortino, "CVD diamond particle detectors used as on-line dosimeters in clinical radiotherapy," Nucl. Instrum. Methods Phys. Res. A 518, 421-422 (2004).

${ }^{48}$ C. M. Buttar, J. Conway, R. Meyfarth, G. Scarsbrook, P. J. Sellin, and A. Whitehead, "CVD diamond detectors as dosimeters for radiotherapy," Nucl. Instrum. Methods Phys. Res. A 392, 281-284 (1997).

${ }^{49}$ G. Cuttone, L. Azario, L. B. Tonghi, E. Borchi, D. Boscarino, M. Bruzzi, M. Bucciolini, G. A. P. Cirrone, C. De Angelis, G. Della Mea, P. Fattibene, C. Gori, A. Guasti, S. Maggioni, S. Mazzocchi, S. Onori, M. Pacilio, E. Petetti, A. Piermattei, S. Pirollo, A. Quaranta, L. Raffaele, V. Rigato, A. Rovelli, M. G. Sabini, S. Sciortino, and G. Zatelli, "The CANDIDO project: Development of a CVD diamond dosimeter for applications in radiotherapy," Nucl. Phys. B, Proc. Suppl. 78, 587-591 (1999).

${ }^{50}$ A. Fidanzio, L. Azario, R. Kalish, Y. Avigal, G. Conte, and P. Ascarelli, "A preliminary dosimetric characterization of chemical vapor deposition diamond detector prototypes in photon and electron radiotherapy beams," Med. Phys. 32, 389-395 (2005).

${ }^{51}$ M. J. Guerrero, D. Tromson, P. Bergonzo, and R. Barrett, "Investigation of defects in CVD diamond: Influence for radiotherapy applications," Nucl. Instrum. Methods Phys. Res. A 552, 105-111 (2005).

${ }^{52}$ M. Jung, J. Morel, and P. Siffert, "Real-time high intensity x-ray dosimetry diamond monitors: Response simulations compared to silicon sensitivities," Nucl. Instrum. Methods Phys. Res. A 554, 514-526 (2005).

${ }^{53} \mathrm{C}$. Manfredotti, "CVD diamond detectors for nuclear and dosimetric applications," Diamond Relat. Mater. 14, 531-540 (2005).

${ }^{54}$ A. J. Whitehead, R. Airey, C. M. Buttar, J. Conway, G. Hill, S. Ramkumar, G. A. Scarsbrook, R. S. Sussmann, and S. Walker, "CVD diamond for medical dosimetry applications," Nucl. Instrum. Methods Phys. Res. A 460, 20-26 (2001).

${ }^{55}$ C. Burman, C. S. Chui, G. Kutcher, S. Leibel, M. Zelefsky, T. LoSasso, S. Spirou, Q. Wu, J. Yang, J. Stein, R. Mohan, Z. Fuks, and C. C. Ling, "Planning, delivery, and quality assurance of intensity-modulated radiotherapy using dynamic multileaf collimator: A strategy for large-scale implementation for the treatment of carcinoma of the prostate," Int. J. Radiat. Oncol., Biol., Phys. 39, 863-873 (1997).

${ }^{56}$ D. A. Low, K. S. Chao, S. Mutic, R. L. Gerber, C. A. Perez, and J. A. Purdy, "Quality assurance of serial tomotherapy for head and neck patient treatments," Int. J. Radiat. Oncol., Biol., Phys. 42, 681-692 (1998).

${ }^{57}$ H. Parsai, M. H. Phillips, P. S. Cho, H. Kippenes, P. Gavin, and D. Axen, "Verification of dynamic intensity-modulated beam deliveries in canine subjects," Med. Phys. 28, 2198-2208 (2001).

${ }^{58}$ H. A. Al-Hallaq, C. S. Reft, and J. C. Roeske, "The dosimetric effects of tissue heterogeneities in intensity-modulated radiation therapy (IMRT) of the head and neck," Phys. Med. Biol. 51, 1145-1156 (2006).

${ }^{59}$ P. Cadman, R. Bassalow, N. P. Sidhu, G. Ibbott, and A. Nelson, "Dosimetric considerations for validation of a sequential IMRT process with a commercial treatment planning system," Phys. Med. Biol. 47, 3001-3010 (2002).

${ }^{60}$ P. E. Engström, P. Haraldsson, T. Landberg, H. Sand Hansen, S. Aage Engelholm, and H. Nyström, "In vivo dose verification of IMRT treated head and neck cancer patients," Acta Oncol. 44, 572-578 (2005).

${ }^{61}$ E. Gershkevitsh, C. H. Clark, J. Staffurth, D. P. Dearnaley, and K. R. Trott, "Dose to bone marrow using IMRT techniques in prostate cancer patients," Strahlenther. Onkol. 181, 172-178 (2005).

${ }^{62}$ P. Haraldsson, T. Knoos, H. Nystrom and P. Engstrom, "Monte Carlo study of TLD measurements in air cavities," Phys. Med. Biol. 48, N253N259 (2003).

${ }^{63}$ N. Linthout, D. Verellen, S. Van Acker, M. De Cock, and G. Storme, "Dosimetric evaluation of partially overlapping intensity modulated beams using dynamic mini-multileaf collimation," Med. Phys. 30, 846855 (2003).

${ }^{64}$ D. A. Low, J. F. Dempsey, R. Venkatesan, S. Mutic, J. Markman, E. Mark Haacke, and J. A. Purdy, "Evaluation of polymer gels and MRI as a 3-D dosimeter for intensity-modulated radiation therapy," Med. Phys. 26, 1542-1551 (1999).

${ }^{65}$ D. A. Low, R. L. Gerber, S. Mutic, and J. A. Purdy, "Phantoms for IMRT dose distribution measurement and treatment verification," Int. J. Radiat. Oncol., Biol., Phys. 40, 1231-1235 (1998).

${ }^{66}$ T. Pawlicki, G. Luxton, Q. T. Le, D. Findley, and C. M. Ma, "Lens dose in MLC-based IMRT treatments of the head and neck," Int. J. Radiat. Oncol., Biol., Phys. 59, 293-299 (2004).

${ }^{67}$ I. A. Popescu, C. P. Shaw, S. F. Zavgorodni, and W. A. Beckham, "Absolute dose calculations for Monte Carlo simulations of radiotherapy beams," Phys. Med. Biol. 50, 3375-3392 (2005).

${ }^{68}$ S. L. Richardson, W. A. Tome, N. P. Orton, T. R. McNutt, and B. R. Paliwal, "IMRT delivery verification using a spiral phantom," Med. Phys. 30, 2553-2558 (2003).

${ }^{69} \mathrm{~F}$. Bagne, "A comprehensive study of LiF TL response to high energy photons and electrons," Radiology 123, 753-760 (1977).

${ }^{70}$ J. Dalgleish, "Letter: TLD system for radiotherapy monitoring," Phys. Med. Biol. 18, 465-467 (1973).

${ }^{71}$ M. J. Rossiter, "The use of precision thermoluminescence dosimetry for intercomparison of absorbed dose," Phys. Med. Biol. 20, 735-746 (1975).

${ }^{72}$ B. I. Ruden, "Evaluation of the clinical use of TLD," Acta Radiol. Ther. Phys. Biol. 15, 447-464 (1976).

${ }^{73}$ M. S. Tarakanath and J. Novotny, "Thermoluminescence dosimetry in clinical radiation dose measurements," Strahlentherapie 152, 71-77 (1976).

${ }^{74}$ A. J. Troncalli and J. Chapman, "TLD linearity vs. beam energy and modality," Med. Dosim. 27, 295-296 (2002).

${ }^{75}$ F. M. Khan, K. P. Doppke, K. R. Hogstrom, G. J. Kutcher, R. Nath, S. C. Prasad, J. A. Purdy, M. Rozenfeld, and B. L. Werner, "Clinical electronbeam dosimetry: Report of AAPM Radiation Therapy Committee Task Group No. 25," Med. Phys. 18, 73-109 (1991).

${ }^{76}$ J. Balog, D. Lucas, C. DeSouza, and R. Crilly, "Helical tomotherapy radiation leakage and shielding considerations," Med. Phys. 32, 710-719 (2005).

${ }^{77}$ J. F. Fowler, J. S. Welsh, and S. P. Howard, "Loss of biological effect in prolonged fraction delivery," Int. J. Radiat. Oncol., Biol., Phys. 59, 242 249 (2004).

${ }^{78}$ S. F. Kry, M. Salehpour, D. S. Followill, M. Stovall, D. A. Kuban, R. A. White, and I. I. Rosen, "The calculated risk of fatal secondary malignancies from intensity-modulated radiation therapy," Int. J. Radiat. Oncol., Biol., Phys. 62, 1195-1203 (2005).

${ }^{79}$ E. A. Miles, C. H. Clark, M. T. Urbano, M. Bidmead, D. P. Dearnaley, K. J. Harrington, R. A'Hern, and C. M. Nutting, "The impact of introducing intensity modulated radiotherapy into routine clinical practice," Radiother. Oncol. 77, 241-246 (2005).

${ }^{80}$ F. Sterzing, M. W. Munter, M. Schafer, P. Haering, B. Rhein, C. Thilmann, and J. Debus, "Radiobiological investigation of dose-rate effects in intensity-modulated radiation therapy," Strahlenther. Onkol. 181, 42-48 (2005).

${ }^{81}$ J. Z. Wang, X. A. Li, W. D. D'Souza, and R. D. Stewart, "Impact of prolonged fraction delivery times on tumor control: A note of caution for intensity-modulated radiation therapy (IMRT)," Int. J. Radiat. Oncol., Biol., Phys. 57, 543-552 (2003).

${ }^{82}$ F. García-Vicente, J. M. Delgado, and C. Peraza, "Experimental determination of the convolution kernel for the study of the spatial response of a detector," Med. Phys. 25, 202-207 (1998).

${ }^{83}$ G. Bednarz, M. Saiful Huq, and U. F. Rosenow, "Deconvolution of detector size effect for output factor measurement for narrow Gamma Knife radiosurgery beams," Phys. Med. Biol. 47, 3643-3649 (2002).

${ }^{84}$ D. A. Low, J. F. Dempsey, J. Markman, S. Mutic, E. E. Klein, J. W. Sohn, and J. A. Purdy, "Toward automated quality assurance for intensitymodulated radiation therapy," Int. J. Radiat. Oncol., Biol., Phys. 53, 443452 (2002).

${ }^{85}$ A. Mack, H. Czempiel, H. J. Kreiner, G. Durr, and B. Wowra, "Quality assurance in stereotactic space. A system test for verifying the accuracy of aim in radiosurgery," Med. Phys. 29, 561-568 (2002).

${ }^{86}$ W. L. McLaughlin, C. G. Soares, J. A. Sayeg, E. C. McCullough, R. W. Kline, A. Wu, and A. H. Maitz, "The use of a radiochromic detector for the determination of stereotactic radiosurgery dose characteristics," Med. Phys. 21, 379-388 (1994).

${ }^{87}$ R. Ramani, A. W. Lightstone, D. L. Mason, and P. F. O’Brien, “The use of radiochromic film in treatment verification of dynamic stereotactic radiosurgery," Med. Phys. 21, 389-392 (1994).

${ }^{88} \mathrm{H}$. Shiomi, T. Inoue, S. Nakamura, and T. Inoue, "Quality assurance for an image-guided frameless radiosurgery system using radiochromic film," Radiat. Med. 18, 107-113 (2000).

${ }^{89}$ T. Yasuda, J. Beatty, P. J. Biggs, and K. Gall, "Two-dimensional dose distribution of a miniature x-ray device for stereotactic radiosurgery," Med. Phys. 25, 1212-1216 (1998). 
${ }^{90}$ M. R. Arnfield, K. Otto, V. R. Aroumougame, and R. D. Alkins, “The use of film dosimetry of the penumbra region to improve the accuracy of intensity modulated radiotherapy," Med. Phys. 32, 12-18 (2005).

${ }^{91}$ J. C. L. Chow, B. Wettlaufer, and R. Q. Jiang, "Dosimetric effects on the penumbra region of irregular multi-leaf collimated fields," Phys. Med. Biol. 51, N31-N38 (2006).

${ }^{92}$ S. Agostinelli, S. Garelli, M. Piergentili, and F. Foppiano, "Response to high-energy photons of PTW31014 PinPoint ion chamber with a central aluminum electrode," Med. Phys. 35, 3293-3301 (2008).

${ }^{93}$ S. Pai, I. J. Das, J. F. Dempsey, K. L. Lam, T. J. Losasso, A. J. Olch, J. R. Palta, L. E. Reinstein, D. Ritt, and E. E. Wilcox, "TG-69: Radiographic film for megavoltage beam dosimetry," Med. Phys. 34, 2228-2258 (2007).

${ }^{94}$ T. M. Bogucki, W. R. Murphy, C. W. Baker, S. S. Piazza, and A. G. Haus, "Processor quality control in laser imaging systems," Med. Phys. 24, 581-584 (1997).

${ }^{95}$ A. Niroomand-Rad, C. R. Blackwell, B. M. Coursey, K. P. Gall, J. M. Galvin, W. L. McLaughlin, A. S. Meigooni, R. Nath, J. E. Rodgers, and C. G. Soares, "Radiochromic film dosimetry: Recommendations of AAPM Radiation Therapy Committee Task Group 55. American Association of Physicists in Medicine," Med. Phys. 25, 2093-2115 (1998).

${ }^{96}$ M. J. Butson, T. Cheung, and P. K. Yu, "Spatial resolution of a stacked radiochromic film dosimeter," Radiother. Oncol. 61, 211-213 (2001).

${ }^{97}$ M. J. Butson, J. N. Mathur, and P. E. Metcalfe, "Radiochromic film as a radiotherapy surface-dose detector," Phys. Med. Biol. 41, 1073-1078 (1996).

${ }^{98}$ T. Cheung, M. J. Butso, and P. K. Yu, "Use of multiple layers of Gafchromic film to increase sensitivity," Phys. Med. Biol. 46, N235-N240 (2001).

${ }^{99}$ J. F. Dempsey, D. A. Low, S. Mutic, J. Markman, A. S. Kirov, G. H. Nussbaum, and J. F. Williamson, "Validation of a precision radiochromic film dosimetry system for quantitative two-dimensional imaging of acute exposure dose distributions," Med. Phys. 27, 2462-2475 (2000).

${ }^{100}$ P. O. Kellermann, A. Ertl, and E. Gornik, "A new method of readout in radiochromic film dosimetry," Phys. Med. Biol. 43, 2251-2263 (1998).

${ }^{101}$ L. E. Reinstein and G. R. Gluckman, "Comparison of dose response of radiochromic film measured with $\mathrm{He}-\mathrm{Ne}$ laser, broadband, and filtered light densitometers," Med. Phys. 24, 1531-1533 (1997).

${ }^{102}$ L. E. Reinstein, G. R. Gluckman, and H. I. Amols, "Predicting optical densitometer response as a function of light source characteristics for radiochromic film dosimetry,” Med. Phys. 24, 1935-1942 (1997).

${ }^{103}$ L. E. Reinstein, G. R. Gluckman, and A. G. Meek, "A rapid colour stabilization technique for radiochromic film dosimetry," Phys. Med. Biol. 43, 2703-2708 (1998).

${ }^{104}$ M. J. Butson, T. Cheung, and P. K. Yu, "Absorption spectra variations of EBT radiochromic film from radiation exposure," Phys. Med. Biol. 50, N135-N140 (2005).

${ }^{105}$ S. A. Dini, R. A. Koona, J. R. Ashburn, and A. S. Meigoonia, "Dosimetric evaluation of GAFCHROMIC XR type T and XR type R films," J. Appl. Clin. Med. Phys. 6, 114-134 (2005).

${ }^{106}$ T. Wiezorek, N. Banz, M. Schwedas, M. Scheithauer, H. Salz, D. Georg, and T. G. Wendt, "Dosimetric quality assurance for intensity-modulated radiotherapy feasibility study for a filmless approach," Strahlenther. Onkol. 181, 468-474 (2005).

${ }^{107}$ S. T. Chiu-Tsao, Y. Ho, R. Shankar, L. Wang, and L. B. Harrison, "Energy dependence of response of new high sensitivity radiochromic films for megavoltage and kilovoltage radiation energies," Med. Phys. 32, 33503354 (2005).

${ }^{108}$ T. Cheung, M. J. Butson, and P. K. Yu, "Experimental energy response verification of XR type T radiochromic film," Phys. Med. Biol. 49, N371N376 (2004).

${ }^{109}$ M. J. Butson, P. K. Yu, T. Cheung, and P. Metcalfe, "High sensitivity radiochromic film dose comparisons," Phys. Med. Biol. 47, N291-N295 (2002).

${ }^{110}$ M. J. Butson, P. K. Yu, T. Cheung, and D. Inwood, "Polarization effects on a high-sensitivity radiochromic film," Phys. Med. Biol. 48, N207N211 (2003).

${ }^{111}$ T. Cheung, M. J. Butson, and P. K. Yu, "Post-irradiation colouration of Gafchromic EBT radiochromic film," Phys. Med. Biol. 50, N281-N285 (2005).

${ }^{112}$ M. J. Butson, T. Cheung, and P. K. Yu, "Visible absorption properties of radiation exposed XR type-T radiochromic film," Phys. Med. Biol. 49, N347-N351 (2004).
${ }^{113}$ B. D. Lynch, J. Kozelka, M. K. Ranade, J. G. Li, W. E. Simon, and J. F. Dempsey, "Important considerations for radiochromic film dosimetry with flatbed CCD scanners and EBT GAFCHROMIC film," Med. Phys. 33, 4551-4556 (2006).

${ }^{114}$ C. G. Soares, "New developments in radiochromic film dosimetry," Radiat. Prot. Dosim. 120, 100-106 (2006).

${ }^{115}$ M. Bazioglou and J. Kalef-Ezra, "Dosimetry with radiochromic films: A document scanner technique, neutron response, applications," Appl. Radiat. Isot. 55, 339-345 (2001).

${ }^{116}$ L. Paelinck, W. De Neve, and C. De Wagter, "Precautions and strategies in using a commercial flatbed scanner for radiochromic film dosimetry," Phys. Med. Biol. 52, 231-242 (2007).

${ }^{117}$ J. E. Bayouth, D. Wendt, and S. M. Morrill, "MLC quality assurance techniques for IMRT applications," Med. Phys. 30, 743-750 (2003).

${ }^{118}$ M. Bucciolini, F. B. Buonamici, and M. Casati, "Verification of IMRT fields by film dosimetry," Med. Phys. 31, 161-168 (2004).

${ }^{119}$ N. L. Childress, L. Dong, and I. I. Rosen, "Rapid radiographic film calibration for IMRT verification using automated MLC fields," Med. Phys. 29, 2384-2390 (2002).

${ }^{120}$ S. Gillis, C. De Wagter, J. Bohsung, B. Perrin, P. Williams, and B. J. Mijnheer, "An inter-centre quality assurance network for IMRT verification: Results of the ESTRO QUASIMODO project," Radiother. Oncol. 76, 340-353 (2005).

${ }^{121}$ A. J. Olch, "Dosimetric performance of an enhanced dose range radiographic film for intensity-modulated radiation therapy quality assurance," Med. Phys. 29, 2159-2168 (2002).

${ }^{122} \mathrm{P}$. Tangboonduangjit, I. Wu, M. Butson, A. Rosenfeld, and P. Metcalfe, "Intensity modulated radiation therapy: Film verification of planar dose maps," Australas. Phys. Eng. Sci. Med. 26, 194-199 (2003).

${ }^{123}$ P. Winkler, B. Zurl, H. Guss, P. Kindl, and G. Stuecklschweiger, "Performance analysis of a film dosimetric quality assurance procedure for IMRT with regard to the employment of quantitative evaluation methods," Phys. Med. Biol. 50, 643-654 (2005).

${ }^{124}$ L. Xing and J. G. Li, "Computer verification of fluence map for intensity modulated radiation therapy," Med. Phys. 27, 2084-2092 (2000).

${ }^{125}$ Y. Yan, N. Papanikolaou, X. Weng, J. Penagaricano, and V. Ratanatharathorn, "Fast radiographic film calibration procedure for helical tomotherapy intensity modulated radiation therapy dose verification," Med. Phys. 32, 1566-1570 (2005).

${ }^{126}$ Y. Yang and L. Xing, "Using the volumetric effect of a finite-sized detector for routine quality assurance of multileaf collimator leaf positioning," Med. Phys. 30, 433-441 (2003).

${ }^{127}$ X. R. Zhu, P. A. Jursinic, D. F. Grimm, F. Lopez, J. J. Rownd, and M. T. Gillin, "Evaluation of Kodak EDR2 film for dose verification of intensity modulated radiation therapy delivered by a static multileaf collimator," Med. Phys. 29, 1687-1692 (2002).

${ }^{128}$ J. Esthappan, S. Mutic, W. B. Harms, J. F. Dempsey, and D. A. Low, "Dosimetry of therapeutic photon beams using an extended dose range film," Med. Phys. 29, 2438-2445 (2002).

${ }^{129}$ X. R. Zhu, S. Yoo, P. A. Jursinic, D. F. Grimm, F. Lopez, J. J. Rownd, and M. T. Gillin, "Characteristics of sensitometric curves of radiographic films," Med. Phys. 30, 912-919 (2003).

${ }^{130}$ N. L. Childress and I. I. Rosen, "Effect of processing time delay on the dose response of Kodak EDR2 film," Med. Phys. 31, 2284-2288 (2004).

${ }^{131}$ E. Y. Hirata, C. Cunningham, J. A. Micka, H. Keller, M. W. Kissick, and L. A. DeWerd, "Low dose fraction behavior of high sensitivity radiochromic film," Med. Phys. 32, 1054-1060 (2005).

${ }^{132}$ A. Mack, G. Mack, D. Weltz, S. G. Scheib, H. D. Bottcher, and V. Seifert, "High precision film dosimetry with GAFCHROMIC films for quality assurance especially when using small fields," Med. Phys. 30, 2399-2409 (2003).

${ }^{133}$ J. W. Sohn, J. F. Dempsey, T. S. Suh, and D. A. Low, "Analysis of various beamlet sizes for IMRT with 6 MV photons," Med. Phys. 30, 2432-2439 (2003).

${ }^{134}$ O. A. Zeidan, J. G. Li, D. A. Low, and J. F. Dempsey, "Comparison of small photon beams measured using radiochromic and silver-halide films in solid water phantoms," Med. Phys. 31, 2730-2737 (2004).

${ }^{135}$ M. A. Bazioglou, J. Kalef-Ezra, and C. Kappas, "Comparison of dosimetric techniques for the assessment of basic dosimetric data of stereotactic fields," Phys. Med. Biol. 17, 123-128 (2001).

${ }^{136}$ P. Francescon, S. Cora, P. Scalchi, and F. Colombo, "Use of GAFCHROMIC(TM) film MD-55 and of a new microparallel-plate chamber in the dosimetry of small fields," Phys. Med. Biol. 13, 91-99 (1997). 
${ }^{137}$ A. Somigliana, G. M. Cattaneo, C. Fiorino, S. Borelli, A. del Vecchio, G. Zonca, E. Pignoli, G. Loi, R. Calandrino, and R. Marchesini, "Dosimetry of Gamma Knife and linac-based radiosurgery using radiochromic and diode detectors," Phys. Med. Biol. 44, 887-897 (1999).

${ }^{138}$ P. A. Jursinic and B. E. Nelms, "A 2-D diode array and analysis software for verification of intensity modulated radiation therapy delivery," Med. Phys. 30, 870-879 (2003).

${ }^{139}$ K. M. Langen, S. L. Meeks, D. O. Poole, T. H. Wagner, T. R. Willoughby, O. A. Zeidan, P. A. Kupelian, K. J. Ruchala, and G. H. Olivera, "Evaluation of a diode array for QA measurements on a helical tomotherapy unit," Med. Phys. 32, 3424-3430 (2005).

${ }^{140}$ D. Letourneau, M. Gulam, D. Yan, M. Oldham, and J. W. Wong, "Evaluation of a 2D diode array for IMRT quality assurance," Radiother. Oncol. 70, 199-206 (2004)

${ }^{141}$ J. G. Li, J. F. Dempsey, L. Ding, C. Liu, and J. R. Palta, "Validation of dynamic MLC-controller log files using a two-dimensional diode array," Med. Phys. 30, 799-805 (2003).

${ }^{142}$ S. Amerio, A. Boriano, F. Bourhaleb, R. Cirio, M. Donetti, A. Fidanzio, E. Garelli, S. Giordanengo, E. Madon, F. Marchetto, U. Nastasi, C. Peroni, A. Piermattei, C. J. Sanz Freire, A. Sardo, and E. Trevisiol, "Dosimetric characterization of a large area pixel-segmented ionization chamber," Med. Phys. 31, 414-420 (2004).

${ }^{143}$ J. Pardo, L. Franco, F. Gomez, A. Iglesias, A. Pazos, J. Pena, R. Lobato, J. Mosquera, M. Pombar, and J. Sendon, "Development and operation of a pixel segmented liquid-filled linear array for radiotherapy quality assurance," Phys. Med. Biol. 50, 1703-1716 (2005).

${ }^{144}$ E. Spezi, A. L. Angelini, F. Romani, and A. Ferri, "Characterization of a 2D ion chamber array for the verification of radiotherapy treatments," Phys. Med. Biol. 50, 3361-3373 (2005).

${ }^{145}$ B. Poppe, A. Blechschmnidt, A. Djouguela, R. Kollhoff, A. Rubach, K. C. Willborn, and D. Harder, "Two-dimensional ionization chamber arrays for IMRT plan verification,” Med. Phys. 33, 1005-1015 (2006).

${ }^{146}$ B. Poppe, P. Mehran, R. Kollhoff, and A. Rubach, "Use of a twodimensional ionization chamber array for quality assurance in medical linear accelerators," Z. Med. Phys. 13, 115-122 (2003).

${ }^{147}$ M. Sonoda, M. Takano, J. Miyahara, and H. Kato, "Computed radiography utilizing scanning laser stimulated luminescence," Radiology 148, 833-838 (1983).

${ }^{148}$ A. J. Olch, "Evaluation of a computed radiography system for megavoltage photon beam dosimetry," Med. Phys. 32, 2987-2999 (2005).

${ }^{149}$ M. A. Bazioglou, K. Theodorou, C. Kappas, and J. Kalef-Ezra, "A multipurpose head phantom for stereotactic radiotherapy," Phys. Med. Biol. 18, 121-127 (2002).

${ }^{150}$ C. M. Ma, S. B. Jiang, T. Pawlicki, Y. Chen, J. S. Li, J. Deng, and A. L. Boyer, "A quality assurance phantom for IMRT dose verification," Phys. Med. Biol. 48, 561-572 (2003).

${ }^{151}$ B. Paliwal, W. Tome, S. Richardson, and T. R. Makie, "A spiral phantom for IMRT and tomotherapy treatment delivery verification," Med. Phys. 27, 2503-2507 (2000).

${ }^{152} \mathrm{~A}$. Palm and T. LoSasso, "Influence of phantom material and phantom size on radiographic film response in therapy photon beams," Med. Phys. 32, 2434-2442 (2005).

${ }^{153}$ L. Xing, B. Curran, R. Hill, T. Holmes, L. Ma, K. M. Forster, and A. L. Boyer, "Dosimetric verification of a commercial inverse treatment planning system," Phys. Med. Biol. 44, 463-478 (1999).

${ }^{154} \mathrm{~J}$. Balog, T. Holmes, and R. Vaden, "A helical tomotherapy dynamic quality assurance," Med. Phys. 33, 3939-3950 (2006).

${ }^{155}$ M. A. MacKenzie, M. Lachaine, B. Murray, B. G. Fallone, D. Robinson, and G. C. Field, "Dosimetric verification of inverse planned step and shoot multileaf collimator fields from a commercial treatment planning system,” J. Appl. Clin. Med. Phys. 3, 97-109 (2002).

${ }^{156}$ J. S. Tsai, D. E. Wazer, M. N. Ling, J. K. Wu, M. Fagundes, T. DiPetrillo, B. Kramer, M. Koistinen, and M. J. Engler, "Dosimetric verification of the dynamic intensity-modulated radiation therapy of 92 patients," Int. J. Radiat. Oncol., Biol., Phys. 40, 1213-1230 (1998).

${ }^{157}$ D. Verellen, N. Linthout, D. van den Berge, A. Bel, and G. Storme, "Initial experience with intensity-modulated conformal radiation therapy for treatment of the head and neck region," Int. J. Radiat. Oncol., Biol., Phys. 39, 99-114 (1997).

${ }^{158}$ S. E. Burch, K. J. Kearfott, J. H. Trueblood, W. C. Sheils, J. I. Yeo, and C. K. Wang, "A new approach to film dosimetry for high energy photon beams: Lateral scatter filtering," Med. Phys. 24, 775-783 (1997).

${ }^{159}$ I. J. Yeo, A. Beiki-Ardakani, Y. B. Cho, M. Heydarian, T. Zhang, and M.
Islam, "EDR2 film dosimetry for IMRT verification using low-energy photon filters," Med. Phys. 31, 1960-1963 (2004).

${ }^{160}$ W. B. Harms, Sr., D. A. Low, J. W. Wong, and J. A. Purdy, "A software tool for the quantitative evaluation of 3D dose calculation algorithms," Med. Phys. 25, 1830-1836 (1998).

${ }^{161}$ J. Van Dyk, R. B. Barnett, J. E. Cygler, and P. C. Shragge, "Commissioning and quality assurance of treatment planning computers," Int. J. Radiat. Oncol., Biol., Phys. 26, 261-273 (1993).

${ }^{162}$ N. L. Childress and I. I. Rosen, "The design and testing of novel clinical parameters for dose comparison,” Int. J. Radiat. Oncol., Biol., Phys. 56, 1464-1479 (2003).

${ }^{163}$ D. A. Low and J. F. Dempsey, "Evaluation of the gamma dose distribution comparison method," Med. Phys. 30, 2455-2464 (2003).

${ }^{164}$ M. Stock, B. Kroupa, and D. Georg, "Interpretation and evaluation of the gamma index and the gamma index angle for the verification of IMRT hybrid plans," Phys. Med. Biol. 50, 399-411 (2005).

${ }^{165}$ G. J. Budgell, B. A. Perrin, J. H. L. Mott, J. Fairfoul, and R. I. Mackay, "Quantitative analysis of patient-specific dosimetric IMRT verification," Phys. Med. Biol. 50, 103-119 (2005).

${ }^{166}$ K. T. S. Islam, J. F. Dempsey, M. K. Ranade, M. J. Maryanski, and D. A. Low, "Initial evaluation of commercial optical CT-based 3D gel dosimeter," Med. Phys. 30, 2159-2168 (2003).

${ }^{167}$ H. S. Jin, H. Chung, C. Liu, J. Palta, T. S. Suh, and S. Y. Kim, "A novel dose uncertainty model and its application for dose verification," Med. Phys. 32, 1747-1756 (2005).

${ }^{168}$ G. Nicolini, A. Fogliata, and L. Cozzi, "IMRT with the sliding window: Comparison of the static and dynamic methods. Dosimetric and spectral analysis," Radiother. Oncol. 75, 112-119 (2005).

${ }^{169}$ K. Nygaard, O. H. Odland, Y. Kvinnsland, B. Nygaard, J. Heggdal, and L. P. Muren, "Measurements and treatment planning calculations of electron dose distributions below bolus edges," Radiother. Oncol. 74, 217-220 (2005).

${ }^{170}$ N. Sakthi, P. Keall, I. Mihaylov, Q. W. Wu, Y. Wu, J. F. Williamson, R. Schmidt-Ullrich, and J. V. Siebers, "Monte Carlo-based dosimetry of head-and-neck patients treated with SIB-IMRT," Int. J. Radiat. Oncol., Biol., Phys. 64, 968-977 (2006).

${ }^{171}$ P. Sandilos, A. Angelopoulos, P. Baras, K. Dardoufas, P. Karaiskos, P. Kipouros, M. Kozicki, J. M. Rosiak, L. Sakelliou, I. Seimenis, and L. Vlahos, "Dose verification in clinical IMRT prostate incidents," Int. J. Radiat. Oncol., Biol., Phys. 59, 1540-1547 (2004).

${ }^{172}$ J. Seco, E. Adams, M. Bidmead, M. Partridge, and F. Verhaegen, "Headand-neck IMRT treatments assessed with a Monte Carlo dose calculation engine," Phys. Med. Biol. 50, 817-830 (2005).

${ }^{173}$ S. D. Thomas, M. Mackenzie, G. C. Field, A. M. Syme, and B. G. Fallone, "Patient specific treatment verifications for helical tomotherapy treatment plans," Med. Phys. 32, 3793-3800 (2005).

${ }^{174}$ S. Vedam, A. Docef, M. Fix, M. Murphy, and P. Keall, "Dosimetric impact of geometric errors due to respiratory motion prediction on dynamic multileaf collimator-based four-dimensional radiation delivery," Med. Phys. 32, 1607-1620 (2005).

${ }^{175}$ A. Bakai, M. Alber, and F. Nusslin, "A revision of the gamma-evaluation concept for the comparison of dose distributions," Phys. Med. Biol. 48, 3543-3553 (2003).

${ }^{176}$ A. Hudson, G. Fallone, and C. Field, "A software tool to quantitatively compare dose distributions," Med. Phys. 30, 1952-1952 (2003).

${ }^{177}$ S. B. Jiang, G. C. Sharp, T. Neicu, R. I. Berbeco, S. Flampouri, and T. Bortfeld, "On dose distribution comparison," Phys. Med. Biol. 51, 759776 (2006).

${ }^{178}$ T. Depuydt, A. Van Esch, and D. P. Huyskens, "A quantitative evaluation of IMRT dose distributions: Refinement and clinical assessment of the gamma evaluation," Radiother. Oncol. 62, 309-319 (2002).

${ }^{179}$ J. M. Moran, J. Radawski, and B. A. Fraass, "A dose gradient analysis tool for IMRT QA,” J. Appl. Clin. Med. Phys. 6, 62-73 (2005).

${ }^{180}$ G. J. Budgell, Q. Zhang, R. J. Trouncer, and R. I. Mackay, "Improving IMRT quality control efficiency using an amorphous silicon electronic portal imager," Med. Phys. 32, 3267-3278 (2005).

${ }^{181}$ J. Chang, C. H. Obcemea, J. Sillanpaa, J. Mechalakos, and C. Burman, "Use of EPID for leaf position accuracy QA of dynamic multi-leaf collimator (DMLC) treatment," Med. Phys. 31, 2091-2096 (2004).

${ }^{182}$ W. van Elmpt, L. McDermott, S. Nijsten, M. Wendling, P. Lambin, and B. Mijnheer, "A literature review of electronic portal imaging for radiotherapy dosimetry," Radiother. Oncol. 88, 289-309 (2008).

${ }^{183}$ B. M. McCurdy, K. Luchka, and S. Pistorius, "Dosimetric investigation 
and portal dose image prediction using an amorphous silicon electronic portal imaging device," Med. Phys. 28, 911-924 (2001).

${ }^{184}$ B. M. McCurdy and S. Pistorius, "A two-step algorithm for predicting portal dose images in arbitrary detectors," Med. Phys. 27, 2109-2116 (2000).

${ }^{185}$ A. Van Esch, T. Depuydt, and D. P. Huyskens, "The use of an aSi-based EPID for routine absolute dosimetric pre-treatment verification of dynamic IMRT fields," Radiother. Oncol. 71, 223-234 (2004).

${ }^{186}$ K. Chytyk and B. M. McCurdy, "Comprehensive fluence model for absolute portal dose image prediction,” Med. Phys. 36, 1389-1398 (2009).

${ }^{187}$ Y. Chen, J. M. Moran, D. A. Roberts, Y. El-Mohri, L. E. Antonuk, and B. A. Fraass, "Performance of a direct-detection active matrix flat panel dosimeter (AMFPD) for IMRT measurements," Med. Phys. 34, 49114922 (2007).

${ }^{188}$ Y. El-Mohri, L. E. Antonuk, J. Yorkston, K. W. Jee, M. Maolinbay, K. L. Lam, and J. H. Siewerdsen, "Relative dosimetry using active matrix flatpanel imager (AMFPI) technology," Med. Phys. 26, 1530-1541 (1999).

${ }^{189}$ J. M. Moran, D. A. Roberts, T. S. Nurushev, L. E. Antonuk, Y. El-Mohri, and B. A. Fraass, "An active matrix flat panel dosimeter (AMFPD) for in-phantom dosimetric measurements," Med. Phys. 32, 466-472 (2005).

${ }^{190}$ M. Sabet, F. W. Menk, and P. B. Greer, "Evaluation of an a-Si EPID in direct detection configuration as a water-equivalent dosimeter for transit dosimetry," Med. Phys. 37, 1459-1467 (2010).

${ }^{191}$ L. N. McDermott, M. Wendling, J. J. Sonke, M. van Herk, and B. J. Mijnheer, "Replacing pretreatment verification with in vivo EPID dosimetry for prostate IMRT," Int. J. Radiat. Oncol., Biol., Phys. 67, 15681577 (2007).

${ }^{192}$ L. N. McDermott, M. Wendling, B. van Asselen, J. Stroom, J. J. Sonke, M. van Herk, and B. J. Mijnheer, "Clinical experience with EPID dosimetry for prostate IMRT pre-treatment dose verification," Med. Phys. 33, 3921-3930 (2006).

${ }^{193}$ M. Wendling, R. J. Louwe, L. N. McDermott, J. J. Sonke, M. van Herk, and B. J. Mijnheer, "Accurate two-dimensional IMRT verification using a back-projection EPID dosimetry method," Med. Phys. 33, 259-273 (2006).

${ }^{194}$ J. V. Siebers, J. O. Kim, L. Ko, P. J. Keall, and R. Mohan, "Monte Carlo computation of dosimetric amorphous silicon electronic portal images," Med. Phys. 31, 2135-2146 (2004).

${ }^{195}$ R. M. Howell, I. P. Smith, and C. S. Jarrio, "Establishing action levels for EPID-based QA for IMRT," J. Appl. Clin. Med. Phys. 9, 2721 (2008).

${ }^{196}$ M. D'Andrea, G. Laccarino, S. Carpino, L. Strigari, and M. Benassi, "Primary photon fluence extraction from portal images acquired with an amorphous silicon flat panel detector: Experimental determination of a scatter filter," J. Exp. Clin. Cancer Res. 26, 125-132 (2007).

${ }^{197}$ A. F. Monti and G. Frigerio, "Dosimetric verification of 6 and $18 \mathrm{MV}$ intensity modulated photon beams using a dedicated fluoroscopic electronic portal imaging device (EPID)," Radiother. Oncol. 81, 88-96 (2006).

${ }^{198}$ C. Talamonti, M. Casati, and M. Bucciolini, "Pretreatment verification of IMRT absolute dose distributions using a commercial a-Si EPID," Med. Phys. 33, 4367-4378 (2006).

${ }^{199}$ M. van Zijtveld, M. L. Dirkx, H. C. de Boer, and B. J. Heijmen, "3D dose reconstruction for clinical evaluation of IMRT pretreatment verification with an EPID," Radiother. Oncol. 82, 201-207 (2007).

${ }^{200}$ P. Winkler, A. Hefner, and D. Georg, "Dose-response characteristics of an amorphous silicon EPID," Med. Phys. 32, 3095-3105 (2005).

${ }^{201}$ W. van Elmpt, S. Petit, D. De Ruysscher, P. Lambin, and A. Dekker, "3D dose delivery verification using repeated cone-beam imaging and EPID dosimetry for stereotactic body radiotherapy of non-small cell lung cancer," Radiother. Oncol. 94, 188-194 (2010).

${ }^{202}$ G. S. Ibbott, "QA in radiation therapy: The RPC perspective," J. Phys.: Conf. Ser. 250, 012001 (2010).

${ }^{203}$ M. Oldham, J. H. Siewerdsen, A. Shetty, and D. A. Jaffray, "High resolution gel-dosimetry by optical-CT and MR scanning," Med. Phys. 28, 1436-1445 (2001).

${ }^{204}$ R. G. Kelly, K. J. Jordan, and J. J. Battista, "Optical CT reconstruction of 3D dose distributions using the ferrous-benzoic-xylenol (FBX) gel dosimeter," Med. Phys. 25, 1741-1750 (1998).

${ }^{205}$ P. Guo, J. Adamovics, and M. Oldham, "Characterization of a new radiochromic three-dimensional dosimeter," Med. Phys. 33, 1338-1345 (2006).

${ }^{206}$ M. J. Maryanski, J. C. Gore, R. P. Kennan, and R. J. Schulz, "NMR relaxation enhancement in gels polymerized and cross-linked by ionizing radiation: A new approach to 3D dosimetry by MRI," Magn. Reson. Imaging 11, 253-258 (1993).

${ }^{207}$ I. C. Baustert, M. Oldham, T. A. Smith, C. Hayes, S. Webb, and M. O. Leach, "Optimized MR imaging for polyacrylamide gel dosimetry," Phys. Med. Biol. 45, 847-858 (2000).

${ }^{208}$ Y. De Deene, "How to scan polymer gels with MRI," J. Phys.: Conf. Ser. 250, 012015 (2010).

${ }^{209}$ J. C. Gore, M. Ranade, M. J. Maryanski, and R. J. Schulz, "Radiation dose distributions in three dimensions from tomographic optical density scanning of polymer gels: I. Development of an optical scanner," Phys. Med. Biol. 41, 2695-2704 (1996).

${ }^{210}$ C. Baldock, Y. De Deene, S. Doran, G. Ibbott, A. Jirasek, M. Lepage, K. B. McAuley, M. Oldham, and L. J. Schreimer"Topical Review: Polymer gel dosimetry," Phys. Med. Biol. 55, R1-R63 (2010).

${ }^{211}$ M. Lepage, A. K. Whittaker, L. Rintoul, S. A. Back, and C. Baldock, "Modelling of post-irradiation events in polymer gel dosimeters," Phys. Med. Biol. 46, 2827-2839 (2001).

${ }^{212}$ A. Ertl, A. Berg, M. Zehetmayer, and P. Frigo, "High-resolution dose profile studies based on MR imaging with polymer BANG(TM) gels in stereotactic radiation techniques," Magn. Reson. Imaging 18, 343-349 (2000).

${ }^{213}$ G. S. Ibbott, M. J. Maryanski, P. Eastman, S. D. Holcomb, Y. Zhang, R. G. Avison, M. Sanders, and J. C. Gore, "Three-dimensional visualization and measurement of conformal dose distributions using magnetic resonance imaging of BANG polymer gel dosimeters," Int. J. Radiat. Oncol., Biol., Phys. 38, 1097-1103 (1997).

${ }^{214}$ M. Oldham, G. R. Gluckman, and L. H. Kim, "3D verification of a prostate IMRT treatment by polymer gel-dosimetry and optical-CT scanning," J. Phys.: Conf. Ser. 3, 293-296 (2004).

${ }^{215}$ S. G. Scheib and S. Gianolini, "Three-dimensional dose verification using BANG gel: A clinical example,” J. Neurosurg. 97, 582-587 (2002).

${ }^{216}$ K. Vergote, Y. De Deene, F. Claus, W. De Gersem, B. Van Duyse, L. Paelinck, E. Achten, W. De Neve, and C. De Wagter, "Application of monomer/polymer gel dosimetry to study the effects of tissue inhomogeneities on intensity-modulated radiation therapy (IMRT) dose distributions," Radiother. Oncol. 67, 119-128 (2003).

${ }^{217}$ Y. Xu, C. S. Wuu, and M. J. Maryanski, "Performance of a commercial optical CT scanner and polymer gel dosimeters for 3-D dose verification," Med. Phys. 31, 3024-3033 (2004).

${ }^{218}$ J. C. Gore, Y. S. Kang, and R. J. Schulz, "Measurement of radiation dose distributions by nuclear magnetic resonance (NMR) imaging," Phys. Med. Biol. 29, 1189-1197 (1984).

${ }^{219}$ H. Fricke and E. J. Hart, in Radiation Dosimetry, edited by F. H. Attix and W. C. Roesch (Academic Press, New York, 1966), Vol. 2.

${ }^{220}$ H. Fricke and E. L. Hart, "The chemical action of roentgen rays on dilute ferrosulphate solutions as a measure of dose," Am. J. Roentgenol., Radium Ther. Nucl. Med. 18, 430-432 (1927).

${ }^{221}$ B. J. Balcom, T. J. Lees, A. R. Sharp, N. S. Kulkarni, and G. S. Wagner, "Diffusion in $\mathrm{Fe}(\mathrm{II} / \mathrm{III})$ radiation dosimetry gels measured by magnetic resonance imaging," Phys. Med. Biol. 40, 1665-1676 (1995).

${ }^{222}$ C. Baldock, P. J. Harris, A. R. Piercy, and B. Healy, "Experimental determination of the diffusion coefficient in two-dimensions in ferrous sulphate gels using the finite element method," Australas. Phys. Eng. Sci. Med. 24, 19-30 (2001).

${ }^{223}$ J. Adamovics and M. J. Maryanski, "Characterisation of PRESAGE ${ }^{\mathrm{TM}}$ : A new 3-D radiochromic solid polymer dosimeter for ionising radiation," Radiat. Prot. Dosim. 120, 107-112 (2006)

${ }^{224}$ H. S.Sakhalkar, J. Adamovics, G. Ibbott, and M. Oldham, "A comprehensive evaluation of the PRESAGE optical-CT 3D dosimetry system," Med. Phys. 36, 71-82 (2009).

${ }^{225}$ M. Oldham, "Optical-CT scanning of polymer gels," J. Phys.: Conf. Ser. 3, 122-135 (2004).

${ }^{226} \mathrm{M}$. Oldham and L. Kim, "A study of geometrical and reconstruction artifacts in 3D gel-dosimetry utilizing optical-CT," Med. Phys. 30, 1426 (2003).

${ }^{227}$ M. Oldham and L. Kim, "Optical-CT gel-dosimetry. II: Optical artifacts and geometrical distortion," Med. Phys. 31, 1093-1104 (2004).

${ }^{228}$ Y. De Deene and C. De Wagter, "Artefacts in multi-echo $T_{2}$ imaging for high-precision gel dosimetry: III. Effects of temperature drift during scanning," Phys. Med. Biol. 46, 2697-2711 (2001).

${ }^{229}$ Y. De Deene, C. De Wagter, W. De Neve, and E. Achten, "Artefacts in multi-echo $T_{2}$ imaging for high-precision gel dosimetry: I. Analysis and compensation of eddy currents," Phys. Med. Biol. 45, 1807-1823 (2000). 
${ }^{230}$ Y. De Deene, C. De Wagter, W. De Neve, and E. Achten, "Artefacts in multi-echo $T_{2}$ imaging for high-precision gel dosimetry: II. Analysis of $B_{1}$-field inhomogeneity," Phys. Med. Biol. 45, 1825-1839 (2000).

${ }^{231}$ M. A. Bero, W. B. Gilboy, and P. M. Glover, "An optical method for three-dimensional dosimetry,” J. Radiol. Prot. 20, 287-294 (2000).

${ }^{232}$ S. J. Doran, K. K. Koerkamp, M. A. Bero, P. Jenneson, E. J. Morton, and W. B. Gilboy, “A CCD-based optical CT scanner for high-resolution 3D imaging of radiation dose distributions: Equipment specifications, optical simulations and preliminary results," Phys. Med. Biol. 46, 3191-3213 (2001).

${ }^{233}$ M. J. Maryañski, Y. Z. Zastavker, and J. C. Gore, "Radiation dose distributions in three dimensions from tomographic optical density scanning of polymer gels: II. Optical properties of the BANG polymer gel," Phys. Med. Biol. 41, 2705-2717 (1996).

${ }^{234}$ M. Oldham, J. H. Siewerdsen, S. Kumar, J. Wong, and D. A. Jaffray, "Optical-CT gel-dosimetry I: Basic investigations," Med. Phys. 30, 623634 (2003).

${ }^{235}$ Y. Xu, C. S. Wuu, and M. J. Maryanski, "Determining optimal gel sensitivity in optical CT scanning of gel dosimeters," Med. Phys. 30, 2257-
2263 (2003).

${ }^{236}$ C. S. Wuu and Y. Xu, "Three-dimensional dose verification for intensity modulated radiation therapy using optical CT based polymer gel dosimetry," Med. Phys. 33, 1412-1419 (2006).

${ }^{237}$ T. Olding, O. Holmes, and L. J. Schreiner, "Cone-beam optical computed tomography for gel dosimetry I: Scanner characterizations," Phys. Med. Biol. 55, 2819-2840 (2010).

${ }^{238}$ A. Thomas, M. Pierquet, and M. Oldham, "Achieving accurate radiochromic optical-CT imaging which using a polychromatic light source," J. Phys.: Conf. Ser. 250, 012045 (2010).

${ }^{239}$ K. Jordan, J. Snir, and J. Battista, "Multiple slot array collimator to minimize stray light in optical cone-beam CT," J. Phys.: Conf. Ser. 250, 012062 (2010).

${ }^{240}$ T. Ju, T. Simpson, J. Deasy, and D. Low, "Geometric interpretation of the $\gamma$ distribution comparison technique: Interpolation free calculations," Med. Phys. 35, 879-887 (2008).

${ }^{241}$ D. A. Low, W. B. Harms, S. Mutic, and J. A. Purdy, "A technique for the quantitative evaluation of dose distributions," Med. Phys. 25, 656-661 (1998). 